\title{
Virtual screening of novel compounds as potential ER $\alpha$ inhibitors
}

\author{
Jakkanaboina TilakVijay*, Kandimalla Vivek Babu, Addepally Uma
}

Centre for Biotechnology, Institute of Science \&Technology, Jawaharlal Nehru Technological University, Hyderabad, Telungana, India; J. TilakVijay - E-mail: vijaytilak28@gmail.com

Received April 2, 2019; Accepted April 12, 2019; Published April 30, 2019

DOI: $10.6026 / 97320630015321$

Abstract:

Majority of breast cancers diagnosed today are estrogen receptor (ER)-positive, however, progesterone receptor-positive (PR-positive) is also responsible for breast cancer. Tumors that are ER/PR-positive are much more likely to respond to hormone therapy than tumors that are ER/PR-negative. Nearly $105 \mathrm{ER} \alpha$ inhibitors from literature when docked resulted in 31 compounds (pyrazolo[1,5-a]pyrimidine analogs and chromen-2-one derivatives) with better binding affinities. The maximum score obtained was -175.282 kcal/mol for compound, [2-(4Fluoro-phenylamino)-pyridin-3-yl]-\{4-[2-phenyl-7- (3, 4, 5-trimethoxy-phenyl)-pyrazolo[1,5-a]pyrimidine-5-carbonyl]-piperazin-1-yl\}-methanone. The major H-bond interactions are observed with Thr347. In pursuit to identify novel ER $\alpha$ inhibitory ligands, virtual screening was carried out by docking pyrazole, bipyrazole, thiazole, thiadiazole etc scaffold analogs from literature.34 bipyrazoles from literature revealed Compound 2, ethyl 5-amino-1-(5-amino-3-anilino-4-ethoxycarbonyl-pyrazol-1-yl)-3-anilino-pyrazole-4-carboxylate, with -175.9 kcal/mol binding affinity with the receptor, where a favourable H-bond was formed with Thr347.On the other hand, screening 2035 FDA approved drugs from Drug Bank database resulted in 11 drugs which showed better binding affinities than ER $\alpha$ bound tamoxifen. Consensus scoring using 5 scoring schemes such as Mol Dock score, mcule, SwissDock, Pose\&Rank and DSX respectively resulted in better rank-sumsfor Lomitapide, Itraconazole, Cobicistat, Azilsartanmedoxomil, and Zafirlukast.

Keywords: molecular docking, virtual screening, ER $\alpha$, estrogen, bipyrazoles, drug Bank

\section{Background:}

Majority of breast cancers diagnosed today are estrogen receptor (ER)-positive, where, estrogen binds to estrogen receptors on the surface of the cell [1]. According to the American Cancer Society, about 2 out of every 3 cases of breast cancer is hormone receptorpositive. However, in certain cases, progesterone receptor-positive (PR-positive) is also responsible for breast cancer [2]. Tumors that are ER/PR-positive are much more likely to respond to hormone therapy than tumors that are ER/PR-negative. ER $\alpha$-positive breast cancer is more resistant to chemotherapy than ER $\alpha$-negative cancer [3]. Estrogen- receptor status and outcomes of modern chemotherapy for patients with node-positive breast cancer is known. ER $\alpha$ plays an important role in determining the sensitivity of breast cancer cells to chemotherapeutic agents in vitro [4]. Down regulation of Aurora-A overrides estrogen-mediated growth and chemo resistance in breast cancer cells. Patients with ER- $\alpha$-positive tumors have a slightly better survival rate than patients with ER- $\alpha$ negative. However, both the ER and PR respond to the drug tamoxifen, designed to interfere the function of ER- $\alpha$ [5]. Tamoxifen decreases the incidence of invasive and non-invasive breast cancer. In spite of the tamoxifen administered side effects, its use as a breast cancer preventive agent is appropriate in many women at increased risk for the disease [6]. ER- $\alpha$ is thought to function as a ligand-activated transcription factor. Extracellular signals can also stimulate ER- $\alpha$-mediated transcription in the absence of estrogen. Stimulated ER- $\alpha$ can influence gene expression by associating with other transcription factors without binding directly to DNA 

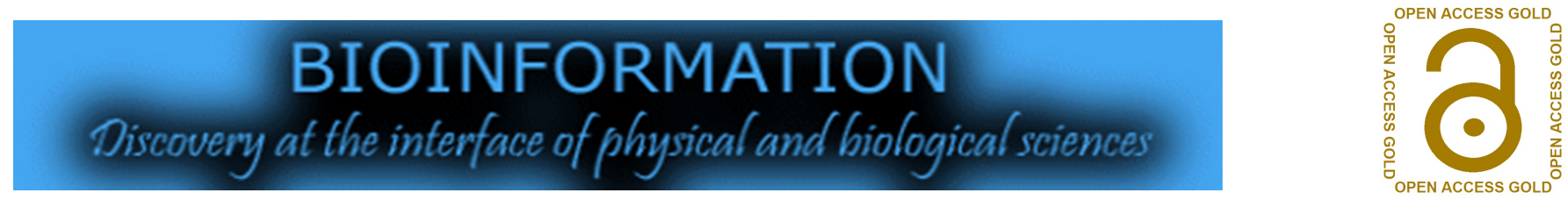

Estrogen receptor alpha rapidly activates the IGF-1 receptor pathway [7-8]. Specific binding sites for estrogen at the outer surfaces of isolated endometrial cells are known. Estrogens stimulate growth of many breast cancer cells. Reducing estrogen levels or blocking often leads to a clinical response in patients with receptor-positive disease. In premenopausal women, estrogen production is high and in postmenopausal women relatively small amounts of estrogens are produced. These low levels of estrogens can be inhibited either by blocking the estrogen receptor, or by inhibiting the peripheral conversion of androgens to estrogens [9]. The most widely accepted pharmacologic endocrine therapies for breast cancer are treatment with anti estrogens [10]. Tamoxifen has been shown to be effective in both premenopausal women as well as in postmenopausal women [11]. Tamoxifen is the most widely used and extensively studied anti estrogen and its role in the management of patients with breast cancer is well established [12]. However, extensive evaluation of tamoxifen treatment revealed significant side effects such as endometrial cancer, blood clots and the development of acquired resistance. Hence, there is a pressing need for the improvement and/or development of new antiestrogens for the prevention and treatment of breast cancer.

\section{Materials \& Methods: \\ Receptor structure for molecular docking:}

A search for Estrogen Receptor alpha (ER- $\alpha$ ) structure in Protein Data Bank (PDB) [www.rcsb.org/pdb] revealed several hits with bound ligands and drugs. In general, the selection of the receptor is based on highest possible resolution, no mutations or modified residues and the presence of bound ligand or drug [13] in particular. The resolution ensures that 3D structures utilized for docking were of a good quality and on the other hand, the structure should be devoid of any mutations, this is because mutations might have profound effects on the final confirmation of a protein [14] [15]. Moreover, a co-crystallized bound ligand represents better geometric orientation within the active site space of the protein. Therefore, the 3D structure of ER $\alpha$ bound with an antagonist, i.e. 4hydroxytamoxifen (PDB ID: 3ERT), was selected as the preferred docking target protein.

\section{Molecular Docking Analysis:}

Molecular docking is a study of non-bonded, non-covalent interactions between a receptor or active site region of a protein and a drug or chemical molecule forming an intermolecular complex [16]. Docking is carried out to dock various conformations of small molecules to a receptor followed by evaluation of the molecules with respect to the geometrical orientation and complementarity in terms of shape and properties, such as electrostatics [17]. The outcome of a docking routine includes affinity prediction (scoring) for the molecules investigated, yielding a relative rank ordering of the docked compounds with respect to affinity, reported as $\mathrm{kcal} / \mathrm{mol}[\mathbf{1 8}]$.

\section{Molegro Virtual Docker:}

Molegro Virtual Docker is an integrated platform for predicting protein - ligand interactions [19]. All default options including preparation of the molecules to determination of the potential binding sites of the target protein, and prediction of the binding modes of the ligands were employed.

\section{Ligand Drawing:}

All ligands were drawn using ISIS/Draw (v. 2.3), which is a userfriendly drawing package that enables to draw chemical structures. ISIS/Draw is mainly a 2D drawing program with structure and reaction validation features and can calculate elementary properties such as formula and molecular weight [20] the 2-D structures are converted into 3-dimensional structures using ProDrug2 server [21].

\section{Datasets:}

\section{Set-1: ERa ligands from literature}

Nearly 105 ligands reported as antagonists of ER $\alpha$ such as benzofurans [22], diphenyl amine analogs [23], sulfoximine-based acyclic triaryl olefins [24], isoxazole derivatives [25] thiazolidinone derivatives [26], tamoxifen mimics [27], pyrazolo[1,5-a]pyrimidine conjugates [28] chromen-2-one derivatives [29] etc. Many of those compounds are serving as anticancer agents [30] antifungal agents [31] and anti-inflammatory agents [32] etc. were selected for molecular docking analysis.

\section{Set-2: ERa Non-tested ligands from literature}

The method employed is to screen similar repertoire of inhibitors reported in various literature sources to identify new probable active compounds, which have not been tested for ER $\alpha$ inhibitory activity. Therefore search initiated for compounds containing pyrazole, bipyrazole, thiazole, thiadiazole etc scaffold analogs reported in Archives of organic chemistry journal www.arkat-usa.org. After preliminary docking investigations, bipyrazole classes of compounds were known to elicit inhibitory characteristics against ER $\alpha$. Hence, a set of 34 bipyrazoles reported in literature www.arkat-usa.org was considered in the study [33-34]. Set-3: Drugs from Drug-Bank Database The rationale to choose Drug Bank database is due to the larger collection and unique resource of drugs with detailed information on each drug and drug target. The latest release of Drug-Bank (version 5.0.10, released 2017-11-14) contains 10,555 drug entries including 1,745 approved small molecule drugs, 877 approved biotech (protein/peptide) drugs, 107 nutraceuticals and over 5,031 experimental drugs. Additionally, 4,775 non-redundant protein (i.e. drug target/enzyme/transporter/carrier) sequences are linked to these drug entries [35]. In the present study, 2035 approved drugs were selected for analysis. 


\section{BIOINFORMATION \\ Discovery at the interface of physical and biological science.}

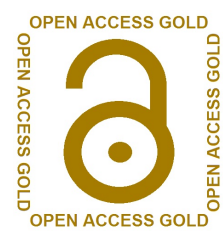

Table 1: Physico-chemical properties and related information of 105 literature compound data

\begin{tabular}{|c|c|c|c|c|c|c|}
\hline Mol Name & SMILES & MW & HBA & HBD & $\log \mathrm{P}$ & RB \\
\hline 1_4d.mol & Oc1ccc(cc1)N(CC1CC1)c1ccc(cc1)O & 255.34 & 2 & 2 & 3.6614 & 4 \\
\hline 6_12.mol & $\mathrm{O}=\mathrm{C} 1 \mathrm{CS}[\mathrm{C} @ \mathrm{H}](\mathrm{N} 1 \mathrm{c} 1 \mathrm{ccccc} 1) \mathrm{c} 1 \mathrm{cccc} 1$ & 255.35 & 1 & 0 & 3.2436 & 2 \\
\hline estradiol.mol & O[C@@H]1CC[C@@H]2[C@@H]1CC[C@@H]1[C@@H]2CCc2cc(ccc21)O & 258.39 & 2 & 2 & 3.5024 & 0 \\
\hline diethylstilbestrol.mol & $\mathrm{CC} / \mathrm{C}(/ \operatorname{c1} 1 \mathrm{ccc}(\operatorname{cc} 1) \mathrm{O})=\mathrm{C}(/ \mathrm{CC}) \backslash \mathrm{c} 1 \mathrm{ccc}(\mathrm{cc} 1) \mathrm{O}$ & 268.38 & 2 & 2 & 4.794 & 5 \\
\hline 1_4e.mol & $\mathrm{CC}(\mathrm{C}) \mathrm{CCN}(\mathrm{c} 1 \mathrm{ccc}(\mathrm{cc} 1) \mathrm{O}) \mathrm{c} 1 \mathrm{ccc}(\mathrm{cc} 1) \mathrm{O}$ & 271.39 & 2 & 2 & 4.4894 & 5 \\
\hline $14 \mathrm{~m} \cdot \mathrm{mol}$ & Oc1ccc(cc1)N(c1cccc1) $11 \operatorname{ccc}(\operatorname{cc} 1) \mathrm{O}$ & 277.34 & 2 & 2 & 4.6332 & 3 \\
\hline 6_1.mol & Oc1ccc(cc1)[C@@H]1SCC $(=\mathrm{O}) \mathrm{N} 1 \mathrm{c1ccc}(\mathrm{cc} 1) \mathrm{O}$ & 287.35 & 3 & 2 & 2.6748 & 2 \\
\hline 1_4j.mol & Oc1cec(cc1) N(Cc1cccec1)c1ccc(cc1)O & 291.37 & 2 & 2 & 4.7281 & 4 \\
\hline 5_2.mol & COc1cc2occ $(\operatorname{cocc} 1 \mathrm{O}) \mathrm{C}(=\mathrm{O}) / \mathrm{C}=\mathrm{C} / \mathrm{c} 1 \mathrm{ccccc} 1$ & 294.32 & 4 & 1 & 3.0895 & 5 \\
\hline 1_4g.mol & Oc1ccc(cc1)N(CC1CCCCC1)c1ccc(cc1)O & 297.43 & 2 & 2 & 4.8503 & 4 \\
\hline $1 \_41 . \mathrm{mol}$ & Oc1ccc(cc1)N(CC1CCCCC1)c1ccc(cc1)O & 297.43 & 2 & 2 & 4.8503 & 4 \\
\hline 6_4.mol & Oc1ccc(cc1)N1[C@@H](SCC1=O)c1ccc(c(c1)O)O & 303.35 & 4 & 3 & 2.3904 & 2 \\
\hline 6 -5.mol & Oc1ccc(cc1)N1[C@@ @H](SCC1=O)c1cc(cc(c1)O)O & 303.35 & 4 & 3 & 2.3904 & 2 \\
\hline 6_6.mol & Oc1ccc(cc1)N1[C@@H](SCC1=O)c1ccc(cc1O)O & 303.35 & 4 & 3 & 2.3904 & 2 \\
\hline 6 11.mol & Cc1ccc(cc1)N1[C@@H](SCC1=O)c1ccc(cc1)Cl & 303.82 & 1 & 0 & 4.2288 & 2 \\
\hline 6_10.mol & Oc1ccc(cc1)N1[C@@H](SCC1=O)c1ccc(cc1)Cl & 305.79 & 2 & 1 & 3.4772 & 2 \\
\hline $14 \mathrm{k} . \mathrm{mol}$ & Oc1ccc(cc1)CN(c1ccc(cc1)O)c1 1 ccc(cc1)O & 307.37 & 3 & 3 & 4.4437 & 4 \\
\hline 1_4h.mol & Oc1ccc(cc1)N(CCC1CCCCC1)c1ccc(cc1)O & 311.46 & 2 & 2 & 5.1743 & 5 \\
\hline $55 . \mathrm{mol}$ & COc1ccc $(\operatorname{cc} 1) \backslash \mathrm{C}=\mathrm{C} \backslash \mathrm{C}(=\mathrm{O}) \mathrm{c} 1 \cos 2 \mathrm{cc}(\mathrm{c}(\mathrm{cc} 21) \mathrm{O}) \mathrm{F}$ & 312.31 & 4 & 1 & 3.229 & 5 \\
\hline 3_vioxx.mol & $\mathrm{CS}(=\mathrm{O})(=\mathrm{O}) \operatorname{c1} \operatorname{ccc}(\operatorname{cc} 1) \mathrm{C} 1=\mathrm{C}(\mathrm{C}(=\mathrm{O}) \mathrm{OC} 1) \operatorname{c} 1 \mathrm{ccccc} 1$ & 314.37 & 4 & 0 & 2.2409 & 3 \\
\hline 5_4.mol & Oc1cc2c $(\operatorname{occ} 2 \mathrm{C}(=\mathrm{O}) / \mathrm{C}=\mathrm{C} / \mathrm{c} 2 \operatorname{cccc}(\mathrm{c} 2) \mathrm{Cl}) \operatorname{cc} 1 \mathrm{~F}$ & 316.72 & 3 & 1 & 3.9997 & 4 \\
\hline 6_13.mol & Cc1ccc(cc1)N1[C@@@H](SCC1=O)c1cccc2ccccs21 & 319.44 & 1 & 0 & 4.713 & 2 \\
\hline 5 - $1 . \mathrm{mol}$ & COc1cc2occ $(\operatorname{coc} 1 \mathrm{C} 1 \mathrm{O}) \mathrm{C}(=\mathrm{O}) \operatorname{c1} \operatorname{cccc}(\mathrm{c} 1) \mathrm{NC}(\mathrm{C})=\mathrm{O}$ & 325.34 & 5 & 2 & 1.5304 & 4 \\
\hline 5_3.mol & COc1cc2occ $(\operatorname{cocc} 1 O) C(=O) / C=C / \operatorname{clcccc}(c 1) \mathrm{Cl}$ & 328.76 & 4 & 1 & 3.6075 & 5 \\
\hline 8_11d.mol & Oc1ccc $(\mathrm{cc} 1) \mathrm{C} 1=\mathrm{C}(\mathrm{c} 2 \operatorname{ccccc} 2) \mathrm{C} 2(\mathrm{OC} 1=\mathrm{O}) \mathrm{C}=\mathrm{CC}(=\mathrm{O}) \mathrm{C}=\mathrm{C} 2$ & 330.35 & 4 & 1 & 2.793 & 2 \\
\hline 6 -7.mol & COc1ccc(c(c1)OC)[C@@H]1SCC $(=\mathrm{O}) \mathrm{N} 1 \mathrm{c} 1 \mathrm{ccc}(\mathrm{cc} 1) \mathrm{O}$ & 331.41 & 4 & 1 & 2.4538 & 4 \\
\hline 6_9.mol & COc1ccc(cc1OC)[C@@ $]$ 1SCC $(=\mathrm{O}) \mathrm{N} 1 \mathrm{c} 1 \mathrm{ccc}(\mathrm{cc} 1) \mathrm{O}$ & 331.41 & 4 & 1 & 2.4538 & 4 \\
\hline 8_11b.mol & Fc1 1 ccc $(\operatorname{cc} 1) \mathrm{C} 1=\mathrm{C}(\mathrm{c} 2 \mathrm{ccccc} 2) \mathrm{C} 2(\mathrm{OC} 1=\mathrm{O}) \mathrm{C}=\mathrm{CC}(=\mathrm{O}) \mathrm{C}=\mathrm{C} 2$ & 332.34 & 3 & 0 & 3.2169 & 2 \\
\hline 4_4m.mol & $\mathrm{CC}(\mathrm{C})(\mathrm{C}) \backslash \mathrm{C}=\mathrm{C} \backslash \mathrm{c} 1 \mathrm{c}($ onc1c1ccc $(\mathrm{cc} 1) \mathrm{O}) \mathrm{c} 1 \mathrm{ccc}(\mathrm{cc} 1) \mathrm{O}$ & 335.43 & 4 & 2 & 5.6743 & 5 \\
\hline 5_7.mol & COc1cc2occ $(\operatorname{c2cc} 1 \mathrm{O}) \mathrm{C}(=\mathrm{O}) / \mathrm{C}=\mathrm{C} / \mathrm{c} 1 \mathrm{ccc} 2 \mathrm{c}(\mathrm{c} 1) \mathrm{OCO} 2$ & 338.33 & 6 & 1 & 2.424 & 5 \\
\hline 8_111.mol & $\mathrm{O}=\mathrm{C} 1 \mathrm{C}=\mathrm{CC} 2(\mathrm{OC}(=\mathrm{O}) \mathrm{C}(=\mathrm{C} 2 \mathrm{c} 2 \mathrm{ccccc} 2) \operatorname{coc} c(\mathrm{cc} 2) \mathrm{C} \# \mathrm{~N}) \mathrm{C}=\mathrm{C} 1$ & 339.36 & 4 & 0 & 2.9424 & 2 \\
\hline 8 11f.mol & COc1 $1 \operatorname{ccc}(\operatorname{cc} 1) \mathrm{C} 1=\mathrm{C}(\operatorname{coc} \operatorname{cccc} 2) \mathrm{C} 2(\mathrm{OC} 1=\mathrm{O}) \mathrm{C}=\mathrm{CC}(=\mathrm{O}) \mathrm{C}=\mathrm{C} 2$ & 344.38 & 4 & 0 & 2.8247 & 3 \\
\hline 8_11g.mol & $\mathrm{OCc} 1 \mathrm{ccc}(\mathrm{ccc} 1) \mathrm{C} 1=\mathrm{C}(\mathrm{c} 2 \mathrm{ccccc} 2) \mathrm{C} 2(\mathrm{OC} 1=\mathrm{O}) \mathrm{C}=\mathrm{CC}(=\mathrm{O}) \mathrm{C}=\mathrm{C} 2$ & 344.38 & 4 & 1 & 2.5421 & 3 \\
\hline 8_11n.mol & $\mathrm{O}=\mathrm{Cc} 1 \mathrm{sc}(\mathrm{cc} 1) \mathrm{C} 1=\mathrm{C}(\mathrm{c} 2 \operatorname{ccccc} 2) \mathrm{C} 2(\mathrm{OC} 1=\mathrm{O}) \mathrm{C}=\mathrm{CC}(=\mathrm{O}) \mathrm{C}=\mathrm{C} 2$ & 348.38 & 4 & 0 & 1.8088 & 3 \\
\hline 3_9b.mol & $\mathrm{CC}(\operatorname{c} 1 \mathrm{ccc}(\mathrm{ccc} 1) \mathrm{S}(\mathrm{C})(\mathrm{N})=\mathrm{O})=\mathrm{C}(\mathrm{c} 1 \mathrm{ccc} c \mathrm{c} 1) \mathrm{c} 1 \mathrm{ccccc} 1$ & 348.51 & 2 & 1 & & 5 \\
\hline 8 11c.mol & $\mathrm{O}=\mathrm{C} 1 \mathrm{OC} 2(\mathrm{C}=\mathrm{CC}(=\mathrm{O}) \mathrm{C}=\mathrm{C} 2) \mathrm{C}(=\mathrm{C} 1 \mathrm{c} 1 \mathrm{oc} 2 \mathrm{ccccc} 2 \mathrm{c} 1) \mathrm{c} 1 \mathrm{ccccc} 1$ & 354.37 & 4 & 0 & 2.7922 & 2 \\
\hline 4_4a.mol & Oc1ccc(cc1)c1onc $(\mathrm{c} 1 / \mathrm{C}=\mathrm{C} / \mathrm{c} 1 \mathrm{ccccc} 1) \mathrm{c} 1 \mathrm{ccc}(\mathrm{cc} 1) \mathrm{O}$ & 355.41 & 4 & 2 & 5.7315 & 5 \\
\hline 8_11k.mol & $\mathrm{O}=\mathrm{C} 1 \mathrm{C}=\mathrm{CC} 2(\mathrm{OC}(=\mathrm{O}) \mathrm{C}(=\mathrm{C} 2 \mathrm{c} 2 \mathrm{ccccc} 2) \mathrm{c} 2 \mathrm{ccc} 3 \mathrm{c}(\mathrm{c} 2) \mathrm{OCO} 3) \mathrm{C}=\mathrm{C} 1$ & 358.36 & 5 & 0 & 2.4119 & 2 \\
\hline $8 \_11 \mathrm{~m} \cdot \mathrm{mol}$ & $\mathrm{OC}(=\mathrm{O}) \operatorname{c} 1 \mathrm{ccc}(\operatorname{cc} 1) \mathrm{C} 1=\mathrm{C}(\mathrm{c} 2 \mathrm{cccc} 2) \mathrm{C} 2(\mathrm{OC} 1=\mathrm{O}) \mathrm{C}=\mathrm{CC}(=\mathrm{O}) \mathrm{C}=\mathrm{C} 2$ & 358.36 & 5 & 1 & 2.7758 & 3 \\
\hline $811 \mathrm{e} \cdot \mathrm{mol}$ & {$[\mathrm{O}-1[\mathrm{~N}+](=\mathrm{O}) \operatorname{c1ccc}(\operatorname{cc} 1) \mathrm{C} 1=\mathrm{C}(\operatorname{coc} \operatorname{ccc} 2) \mathrm{C} 2(\mathrm{OC} 1=\mathrm{O}) \mathrm{C}=\mathrm{CC}(=\mathrm{O}) \mathrm{C}=\mathrm{C} 2$} & 359.35 & 5 & $\frac{1}{0}$ & 3.031 & 2 \\
\hline 6_14.mol & COc1cc(cc(c1OC)OC)[C@@ $@] 1 S C C(=O) N 1 c 1 c c c(c c 1) C$ & 359.47 & 4 & 0 & 2.9527 & 5 \\
\hline $6 \_8 . \mathrm{mol}$ & COc1cc(cc(c1OC)OC)[C@@H]1SCC $(=\mathrm{O}) \mathrm{N} 1 \mathrm{c} 1 \mathrm{ccc}(\mathrm{cc} 1) \mathrm{O}$ & 361.44 & 5 & 1 & 2.2011 & 5 \\
\hline 8 11i.mol & COc1 $\operatorname{ccc}(\operatorname{cc} 1 \mathrm{C} 1=\mathrm{C}(\mathrm{c} 2 \operatorname{ccccc} 2) \mathrm{C} 2(\mathrm{OC} 1=\mathrm{O}) \mathrm{C}=\mathrm{CC}(=\mathrm{O}) \mathrm{C}=\mathrm{C} 2) \mathrm{F}$ & 362.37 & 4 & 0 & 2.9642 & 3 \\
\hline $4 \_4 \mathrm{c} . \mathrm{mol}$ & $\mathrm{Cc} 1 \mathrm{ccc}(\mathrm{cc} 1) \backslash \mathrm{C}=\mathrm{C} \backslash \mathrm{c} 1 \mathrm{c}(\mathrm{onc1} 1 \mathrm{c} 1 \mathrm{ccc}(\mathrm{cc} 1) \mathrm{O}) \mathrm{c} 1 \mathrm{ccc}(\mathrm{cc} 1) \mathrm{O}$ & 369.44 & 4 & 2 & 6.1987 & 5 \\
\hline $13 . \mathrm{mol}$ & Oc1ccc $(\mathrm{cc} 1) \mathrm{C}(\mathrm{c} 1 \mathrm{ccc}(\mathrm{cc} 1) \mathrm{O})=\mathrm{C}(\mathrm{CC}(\mathrm{F})(\mathrm{F}) \mathrm{F}) \mathrm{c} 1 \mathrm{ccccc} 1$ & 370.39 & 2 & 2 & 5.8763 & 5 \\
\hline 5_6.mol & COc1cc2occ $(\operatorname{cocc} 10) \mathrm{C}(=\mathrm{O}) / \mathrm{C}=\mathrm{C} / \mathrm{c} 1 \mathrm{ccc}(\mathrm{cc} 1) \mathrm{c} 1 \mathrm{ccccc} 1$ & 370.42 & 4 & 1 & 4.7739 & 6 \\
\hline 8 11a.mol & $\mathrm{O}=\mathrm{C} 1 \mathrm{OC} 2(\mathrm{C}=\mathrm{CC}(=\mathrm{O}) \mathrm{C}=\mathrm{C} 2) \mathrm{C}(=\mathrm{C} 1 \mathrm{c} 1 \mathrm{sc} 2 \mathrm{ccccc} 2 \mathrm{c} 1) \mathrm{c} 1 \mathrm{ccccc} 1$ & 370.43 & 3 & 0 & 3.1355 & 2 \\
\hline 4_4h.mol & 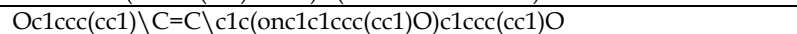 & 371.41 & 5 & 3 & 5.4471 & 5 \\
\hline 4_4i.mol & Oc1ccc(cc1)c1onc $(\mathrm{c} 1 / \mathrm{C}=\mathrm{C} / \mathrm{c} 1 \mathrm{cccc}(\mathrm{c} 1) \mathrm{O}) \mathrm{c} 1 \mathrm{ccc}(\mathrm{cc} 1) \mathrm{O}$ & 371.41 & 5 & 3 & 5.4471 & 5 \\
\hline 4_4d.mol & Oc1ccc(cc1)c1onc(c1/C=C/c1cce(cc1)F)c1ccc(cc1)O & 373.4 & 4 & 2 & 5.871 & 5 \\
\hline 4_4j.mol & CCCCCCC $\backslash \mathrm{C}=\mathrm{C} \backslash \mathrm{c} 1 \mathrm{c}(\mathrm{onc} 1 \mathrm{c} 1 \mathrm{ccc}(\mathrm{cc} 1) \mathrm{O}) \mathrm{c} 1 \mathrm{ccc}(\mathrm{cc} 1) \mathrm{O}$ & 377.52 & 4 & 2 & 6.8921 & 10 \\
\hline hydroxytamoxifen.mol & $\mathrm{CC} \backslash \mathrm{C}(\backslash \mathrm{c} 1 \mathrm{ccccc} 1)=\mathrm{C}(/ \mathrm{c} 1 \mathrm{ccc}(\mathrm{cc} 1) \mathrm{O}) \backslash \mathrm{c} 1 \mathrm{ccc}(\mathrm{cc} 1) \mathrm{OCCN}(\mathrm{C}) \mathrm{C}$ & 387.56 & 3 & 1 & 5.6257 & 9 \\
\hline 4 4e.mol & Oc1 $1 \mathrm{ccc}(\mathrm{cc} 1) \mathrm{c} 1 \mathrm{onc}(\mathrm{c} 1 / \mathrm{C}=\mathrm{C} / \mathrm{c} 1 \mathrm{ccc}(\mathrm{cc} 1) \mathrm{Cl}) \mathrm{c} 1 \mathrm{ccc}(\mathrm{cc} 1) \mathrm{O}$ & 389.85 & 4 & 2 & 6.2495 & 5 \\
\hline 3_2.mol & $\operatorname{CCCCC~}(\operatorname{coccc}(\operatorname{cc} 1) \mathrm{S}(\mathrm{C})(=\mathrm{O})=\mathrm{O})=\mathrm{C}(\mathrm{c} 1 \mathrm{ccccc} 1) \operatorname{c} 1 \mathrm{ccccc} 1$ & 390.57 & 2 & 0 & 6.1213 & 8 \\
\hline 3_9a.mol & $\operatorname{CCCCC~}(\operatorname{c1ccc}(\operatorname{cc} 1) S(C)(N)=O)=C(\operatorname{clccccc} 1) \operatorname{cocccc} 1$ & 390.6 & 2 & 1 & & 8 \\
\hline $4 \_4 \mathrm{k} . \mathrm{mol}$ & CCCCСССC $\backslash \mathrm{C}=\mathrm{C} \backslash \mathrm{c1c}($ onc1c1ccc $(\mathrm{cc} 1) \mathrm{O}) \mathrm{c} 1 \mathrm{ccc}(\mathrm{cc} 1) \mathrm{O}$ & 391.55 & 4 & 2 & 7.2884 & 11 \\
\hline
\end{tabular}




\section{BIOINFORMATION \\ Discevery at the intertace of physical and biological science.}

\begin{tabular}{|c|c|c|c|c|c|c|}
\hline 8_11h.mol & $\mathrm{COc} 1 \mathrm{cc}(\mathrm{cc}(\mathrm{c} 1 \mathrm{OC}) \mathrm{OC}) \mathrm{C} 1=\mathrm{C}(\mathrm{c} 2 \mathrm{ccccc} 2) \mathrm{C} 2(\mathrm{OC} 1=\mathrm{O}) \mathrm{C}=\mathrm{CC}(=\mathrm{O}) \mathrm{C}=\mathrm{C} 2$ & 404.44 & 6 & 0 & 2.3193 & 5 \\
\hline 4_41.mol & $\mathrm{CCCCCCCCC} \backslash \mathrm{C}=\mathrm{C} \backslash \mathrm{c} 1 \mathrm{c}($ onc1c1ccc $(\mathrm{cc} 1) \mathrm{O}) \mathrm{c} 1 \mathrm{ccc}(\mathrm{cc} 1) \mathrm{O}$ & 405.58 & 4 & 2 & 7.6847 & 12 \\
\hline 3_8a.mol & $\operatorname{CCCCC}(\operatorname{c} 1 \operatorname{ccc}(\operatorname{cc} 1) S(\mathrm{C})(=\mathrm{O}) \mathrm{NC} \# \mathrm{~N})=\mathrm{C}(\mathrm{c} 1 \operatorname{ccccc} 1) \operatorname{c} 1 \operatorname{ccccc} 1$ & 415.61 & 3 & 1 & & 9 \\
\hline 4_4f.mol & Oc1ccc(cc1)c1onc(c1/C=C/c1ccc(cc1)C(F)(F)F)c1ccc(cc1)O & 423.41 & 4 & 2 & 6.6143 & 5 \\
\hline 1_4i.mol & Oc1ccc(cc1)N(C[C@@]12C[C@@H]3C[C@@H](C[C@@](Br)(C3)C1)C2)c1ccc(cc1)O & 428.4 & 2 & 2 & 5.3113 & 4 \\
\hline 14_15a.mol & $\mathrm{COc} 1 \mathrm{ccc}(\mathrm{cc} 1) \mathrm{C} 1=\mathrm{C}(\mathrm{Nc} 2 \mathrm{ccc}(\mathrm{cc} 2) \mathrm{OCCN}(\mathrm{C}) \mathrm{C}) \mathrm{c} 2 \operatorname{ccccc} 2 \mathrm{OC} 1=\mathrm{O}$ & 430.54 & 5 & 1 & 3.1602 & 8 \\
\hline 8_11j.mol & $\mathrm{COc} 1 \mathrm{c}(\operatorname{cc}(\mathrm{cc} 1 \mathrm{C} 1=\mathrm{C}(\mathrm{c} 2 \mathrm{cccc} 2) \mathrm{C} 2(\mathrm{OC} 1=\mathrm{O}) \mathrm{C}=\mathrm{CC}(=\mathrm{O}) \mathrm{C}=\mathrm{C} 2) \mathrm{C}) \mathrm{Br}$ & 437.3 & 4 & 0 & 4.0837 & 3 \\
\hline 14_18a.mol & $\mathrm{COc} 1 \mathrm{ccc}(\mathrm{cc} 1) \mathrm{C} 1=\mathrm{C}(\mathrm{Nc} 2 \mathrm{ccc}(\mathrm{cc} 2) \mathrm{OCCN}(\mathrm{C}) \mathrm{C}) \mathrm{c} 2 \mathrm{ccc}(\mathrm{cc} 2 \mathrm{OC} 1=\mathrm{O}) \mathrm{O}$ & 446.54 & 6 & 2 & 2.8758 & 8 \\
\hline 14_15c.mol & $\mathrm{COc} 1 \mathrm{ccc}(\mathrm{cc} 1) \mathrm{C} 1=\mathrm{C}(\mathrm{Nc} 2 \mathrm{ccc}(\mathrm{cc} 2) \mathrm{OCCN} 2 \mathrm{CCCC} 2) \mathrm{c} 2 \mathrm{cccc} 2 \mathrm{OC} 1=\mathrm{O}$ & 456.58 & 5 & 1 & 3.4858 & 8 \\
\hline 14_15b.mol & $\mathrm{CCN}(\mathrm{CC}) \mathrm{CCOc} 1 \mathrm{ccc}(\mathrm{cc} 1) \mathrm{NC} 1=\mathrm{C}(\mathrm{C}(=\mathrm{O}) \mathrm{Oc} 2 \mathrm{cccc} 21) \mathrm{c} 1 \mathrm{ccc}(\mathrm{cc} 1) \mathrm{OC}$ & 458.6 & 5 & 1 & 3.8452 & 10 \\
\hline 14_16a.mol & $\mathrm{COc} 1 \mathrm{ccc}(\mathrm{cc} 1) \mathrm{C} 1=\mathrm{C}(\mathrm{Nc} 2 \mathrm{ccc}(\mathrm{cc} 2) \mathrm{OCCN}(\mathrm{C}) \mathrm{C}) \mathrm{c} 2 \mathrm{ccc}(\mathrm{cc} 2 \mathrm{OC} 1=\mathrm{O}) \mathrm{OC}$ & 460.57 & 6 & 1 & 2.9075 & 9 \\
\hline 14_15d.mol & $\mathrm{COc} 1 \mathrm{ccc}(\mathrm{cc} 1) \mathrm{C} 1=\mathrm{C}(\mathrm{Nc} 2 \mathrm{ccc}(\mathrm{cc} 2) \mathrm{OCCN} 2 \mathrm{CCCCC} 2) \mathrm{c} 2 \mathrm{ccccc} 2 \mathrm{OC} 1=\mathrm{O}$ & 470.61 & 5 & 1 & 3.8821 & 8 \\
\hline 14_15e.mol & $\mathrm{COc} 1 \mathrm{ccc}(\mathrm{cc} 1) \mathrm{C} 1=\mathrm{C}(\mathrm{Nc} 2 \mathrm{ccc}(\mathrm{cc} 2) \mathrm{OCCN} 2 \mathrm{CCOCC} 2) \mathrm{c} 2 \mathrm{ccccc} 2 \mathrm{OC} 1=\mathrm{O}$ & 472.58 & 6 & 1 & 2.8176 & 8 \\
\hline 14_18c.mol & COc1ccc $(\mathrm{cc} 1) \mathrm{C} 1=\mathrm{C}(\mathrm{Nc} 2 \mathrm{ccc}(\mathrm{cc} 2) \mathrm{OCCN} 2 \mathrm{CCCC} 2) \mathrm{c} 2 \mathrm{ccc}(\mathrm{cc} 2 \mathrm{OC} 1=\mathrm{O}) \mathrm{O}$ & 472.58 & 6 & 2 & 3.2014 & 8 \\
\hline 14_18b.mol & $\mathrm{CCN}(\mathrm{CC}) \mathrm{CCOc} 1 \mathrm{ccc}(\mathrm{cc} 1) \mathrm{NC} 1=\mathrm{C}(\mathrm{C}(=\mathrm{O}) \mathrm{Oc} 2 \mathrm{cc}(\mathrm{ccc} 21) \mathrm{O}) \mathrm{c} 1 \mathrm{ccc}(\mathrm{cc} 1) \mathrm{OC}$ & 474.6 & 6 & 2 & 3.5608 & 10 \\
\hline 14_15f.mol & $\mathrm{COc} 1 \mathrm{ccc}(\mathrm{cc} 1) \mathrm{C} 1=\mathrm{C}(\mathrm{Nc} 2 \mathrm{ccc}(\mathrm{cc} 2) \mathrm{OCCN} 2 \mathrm{CCN}(\mathrm{C}) \mathrm{CC} 2) \mathrm{c} 2 \mathrm{ccccc} 2 \mathrm{OC} 1=\mathrm{O}$ & 485.63 & 6 & 1 & 2.9615 & 8 \\
\hline 14_16c.mol & COc1ccc $(\mathrm{cc} 1) \mathrm{C} 1=\mathrm{C}(\mathrm{Nc} 2 \mathrm{ccc}(\mathrm{cc} 2) \mathrm{OCCN} 2 \mathrm{CCCC} 2) \mathrm{c} 2 \mathrm{ccc}(\mathrm{cc} 2 \mathrm{OC} 1=\mathrm{O}) \mathrm{OC}$ & 486.61 & 6 & 1 & 3.2331 & 9 \\
\hline 14_18e.mol & $\mathrm{COc} 1 \mathrm{ccc}(\mathrm{cc} 1) \mathrm{C} 1=\mathrm{C}(\mathrm{Nc} 2 \mathrm{ccc}(\mathrm{cc} 2) \mathrm{OCCN} 2 \mathrm{CCOCC} 2) \mathrm{c} 2 \mathrm{ccc}(\mathrm{cc} 2 \mathrm{OC} 1=\mathrm{O}) \mathrm{O}$ & 488.58 & 7 & 2 & 2.5332 & 8 \\
\hline 14_16b.mol & $\mathrm{CCN}(\mathrm{CC}) \mathrm{CCOc} 1 \mathrm{ccc}(\mathrm{cc} 1) \mathrm{NC} 1=\mathrm{C}(\mathrm{C}(=\mathrm{O}) \mathrm{Oc} 2 \mathrm{cc}(\mathrm{ccc} 21) \mathrm{OC}) \mathrm{c} 1 \mathrm{ccc}(\mathrm{cc} 1) \mathrm{OC}$ & 488.63 & 6 & 1 & 3.5925 & 11 \\
\hline 14_16d.mol & $\mathrm{COc} 1 \mathrm{ccc}(\mathrm{cc} 1) \mathrm{C} 1=\mathrm{C}(\mathrm{Nc} 2 \mathrm{ccc}(\mathrm{cc} 2) \mathrm{OCCN} 2 \mathrm{CCCCC} 2) \mathrm{c} 2 \mathrm{ccc}(\mathrm{cc} 2 \mathrm{OC} 1=\mathrm{O}) \mathrm{OC}$ & 500.64 & 6 & 1 & 3.6294 & 9 \\
\hline 14_18f.mol & $\mathrm{COc} 1 \mathrm{ccc}(\mathrm{cc} 1) \mathrm{C} 1=\mathrm{C}(\mathrm{Nc} 2 \mathrm{ccc}(\mathrm{cc} 2) \mathrm{OCCN} 2 \mathrm{CCN}(\mathrm{C}) \mathrm{CC} 2) \mathrm{c} 2 \mathrm{ccc}(\mathrm{cc} 2 \mathrm{OC} 1=\mathrm{O}) \mathrm{O}$ & 501.63 & 7 & 2 & 2.6771 & 8 \\
\hline 14_16e.mol & COc1ccc(cc1)C1=C(Nc2ccc(cc2)OCCN2CCOCC2)c2ccc(cc2OC1=O)OC & 502.61 & 7 & 1 & 2.5649 & 9 \\
\hline 14_16f.mol & COc1ccc(cc1)C1=C(Nc2ccc(cc2)OCCN2CCN(C)CC2)c2ccc(cc2OC1=O)OC & 515.66 & 7 & 1 & 2.7088 & 9 \\
\hline 11_6a.mol & Fc1ccc(cc1)C1=CC $(=\mathrm{Nc} 2 \mathrm{cc}(\mathrm{nn} 21) \mathrm{c} 1 \mathrm{ccccc} 1) \mathrm{C}(=\mathrm{O}) \mathrm{N} 1 \mathrm{CCN}(\mathrm{CC} 1) \mathrm{C}(=\mathrm{O}) \mathrm{c} 1 \mathrm{cccnc} 1 \mathrm{Nc} 1 \mathrm{ccccc} 1$ & 597.7 & 5 & 1 & 4.5686 & 6 \\
\hline 11_6c.mol & COc1ccc $(\mathrm{cc} 1) \mathrm{C} 1=\mathrm{CC}(=\mathrm{Nc} 2 \mathrm{cc}(\mathrm{nn} 21) \mathrm{c} 1 \mathrm{ccccc} 1) \mathrm{C}(=\mathrm{O}) \mathrm{N} 1 \mathrm{CCN}(\mathrm{CC} 1) \mathrm{C}(=\mathrm{O}) \mathrm{c} 1 \mathrm{ccc} n \mathrm{~N} 1 \mathrm{Nc} 1 \mathrm{ccccc} 1$ & 609.74 & 6 & 1 & 4.1764 & 7 \\
\hline 11_6f.mol & Fc1ccc(cc1)Nc1nccc1C $(=\mathrm{O}) \mathrm{N} 1 \mathrm{CCN}(\mathrm{CC} 1) \mathrm{C}(=\mathrm{O}) \mathrm{C} 1=\mathrm{Nc} 2 \mathrm{cc}(\mathrm{nn} 2 \mathrm{C}(=\mathrm{C} 1) \mathrm{c} 1 \mathrm{ccc}(\mathrm{cc} 1) \mathrm{F}) \mathrm{c} 1 \mathrm{ccccc} 1$ & 615.69 & 5 & 1 & 4.7081 & 6 \\
\hline 11_6h.mol & COc1ccc(cc1)C1=CC $(=\mathrm{Nc} 2 \mathrm{cc}(\mathrm{nn} 21) \mathrm{c} 1 \mathrm{ccccc} 1) \mathrm{C}(=\mathrm{O}) \mathrm{N} 1 \mathrm{CCN}(\mathrm{CC} 1) \mathrm{C}(=\mathrm{O}) \mathrm{c} 1 \mathrm{ccc} n c 1 \mathrm{Nc} 1 \mathrm{ccc}(\mathrm{cc} 1) \mathrm{F}$ & 627.73 & 6 & 1 & 4.3159 & 7 \\
\hline 11_6k.mol & COc1ccc(cc1)Nc1ncccc1C(=O)N1CCN(CC1)C(=O)C1=Nc2cc(nn2C(=C1)c1ccc(cc1)F)c1cccc1 & 627.73 & 6 & 1 & 4.3159 & 7 \\
\hline 11_6d.mol & COc1ccc $(\mathrm{cc} 1 \mathrm{OC}) \mathrm{C} 1=\mathrm{CC}(=\mathrm{Nc} 2 \mathrm{cc}(\mathrm{nn} 21) \mathrm{c} 1 \mathrm{ccccc} 1) \mathrm{C}(=\mathrm{O}) \mathrm{N} 1 \mathrm{CCN}(\mathrm{CC} 1) \mathrm{C}(=\mathrm{O}) \mathrm{c} 1 \mathrm{ccc} n \mathrm{~N} 1 \mathrm{Nc} 1 \mathrm{cccc} 1$ & 639.771 & 7 & 1 & 3.9237 & 8 \\
\hline 11_6m.mol & COc1ccc $(\mathrm{cc} 1) \mathrm{Nc} 1 \mathrm{ncccc} 1 \mathrm{C}(=\mathrm{O}) \mathrm{N} 1 \mathrm{CCN}(\mathrm{CC} 1) \mathrm{C}(=\mathrm{O}) \mathrm{C} 1=\mathrm{Nc} 2 \mathrm{cc}(\mathrm{nn} 2 \mathrm{C}(=\mathrm{C} 1) \mathrm{c} 1 \mathrm{ccc}(\mathrm{cc} 1) \mathrm{OC}) \mathrm{c} 1 \mathrm{ccccc} 1$ & 639.771 & 7 & 1 & 3.9237 & 8 \\
\hline 11_6b.mol & Clc1ccc(cc1Cl)C1=CC (=Nc2cc(nn21)c1ccccc1)C(=O)N1CCN(CC1)C(=O)c1ccenc1Nc1ccccc1 & 648.59 & 5 & 1 & 5.4651 & 6 \\
\hline 11_6i.mol & COc1ccc(cc1OC)C1=CC $(=\mathrm{Nc} 2 \mathrm{cc}(\mathrm{nn} 21) \mathrm{c} 1 \mathrm{ccccc} 1) \mathrm{C}(=\mathrm{O}) \mathrm{N} 1 \mathrm{CCN}(\mathrm{CC} 1) \mathrm{C}(=\mathrm{O}) \mathrm{c} 1 \mathrm{ccc} n c 1 \mathrm{Nc} 1 \mathrm{ccc}(\mathrm{cc} 1) \mathrm{F}$ & 657.76 & 7 & 1 & 4.0632 & 8 \\
\hline 11_6p.mol & COc1ccc(cc1OC)Nc1nccc1C $(=\mathrm{O}) \mathrm{N} 1 \mathrm{CCN}(\mathrm{CC} 1) \mathrm{C}(=\mathrm{O}) \mathrm{C} 1=\mathrm{Nc} 2 \mathrm{cc}(\mathrm{nn} 2 \mathrm{C}(=\mathrm{C} 1) \mathrm{c} 1 \mathrm{ccc}(\mathrm{cc} 1) \mathrm{F}) \mathrm{c} 1 \mathrm{ccccc} 1$ & 657.76 & 7 & 1 & 4.0632 & 8 \\
\hline 11_6n.mol & COc1ccc(cc1)Nc1nccc1C(=O)N1CCN(CC1)C(=O)C1=Nc2cc(nn2C(=C1)c1ccc(c(c1)OC)OC)c1cccc1 & 669.801 & 8 & 1 & 3.671 & 9 \\
\hline 11_6r.mol & $\mathrm{COc1ccc}(\mathrm{cc} 1) \mathrm{C} 1=\mathrm{CC}(=\mathrm{Nc} 2 \mathrm{cc}(\mathrm{nn} 21) \mathrm{c} 1 \mathrm{ccccc} 1) \mathrm{C}(=\mathrm{O}) \mathrm{N} 1 \mathrm{CCN}(\mathrm{CC} 1) \mathrm{C}(=\mathrm{O}) \mathrm{c} 1 \mathrm{cccnc} 1 \mathrm{Nc} 1 \mathrm{ccc}(\mathrm{c}(\mathrm{c} 1) \mathrm{OC}) \mathrm{OC}$ & 669.801 & 8 & 1 & 3.671 & 9 \\
\hline 11_6l.mol & COc1ccc(cc1)Nc1ncccc1C(=O)N1CCN(CC1)C(=O)C1=Nc2cc(nn2C(=C1)c1ccc(c(c1)Cl)Cl)c1cccc1 & 678.62 & 6 & 1 & 5.2124 & 7 \\
\hline 11_6j.mol & COc1cc(cc(c1OC)OC)C1=CC $(=\mathrm{Nc} 2 \mathrm{cc}(\mathrm{nn} 21) \mathrm{c} 1 \mathrm{ccccc} 1) \mathrm{C}(=\mathrm{O}) \mathrm{N} 1 \mathrm{CCN}(\mathrm{CC} 1) \mathrm{C}(=\mathrm{O}) \mathrm{c} 1 \mathrm{cccnc} 1 \mathrm{Nc} 1 \mathrm{ccc}(\mathrm{cc} 1) \mathrm{F}$ & 687.791 & 8 & 1 & 3.8105 & 9 \\
\hline 11_6u.mol & COc1cc(cc(c1OC)OC)Nc1nccc1C $(=\mathrm{O}) \mathrm{N} 1 \mathrm{CCN}(\mathrm{CC} 1) \mathrm{C}(=\mathrm{O}) \mathrm{C} 1=\mathrm{Nc} 2 \mathrm{cc}(\mathrm{nn} 2 \mathrm{C}(=\mathrm{C} 1) \mathrm{c} 1 \mathrm{ccc}(\mathrm{cc} 1) \mathrm{F}) \mathrm{c} 1 \mathrm{cccc} 1$ & 687.791 & 8 & 1 & 3.8105 & 9 \\
\hline 11_6o.mol & COc1ccc(cc1)Nc1ncccc1C(=O)N1CCN(CC1)C(=O)C1=Nc2cc(nn2C(=C1)c1cc(c(c(c1)OC)OC)OC)c1cccc1 & 699.831 & 9 & 1 & 3.4183 & 10 \\
\hline 11_6s.mol & COc1ccc(cc1OC)Nc1ncccc1C $(=\mathrm{O}) \mathrm{N} 1 \mathrm{CCN}(\mathrm{CC} 1) \mathrm{C}(=\mathrm{O}) \mathrm{C} 1=\mathrm{Nc} 2 \mathrm{cc}(\mathrm{nn} 2 \mathrm{C}(=\mathrm{C} 1) \mathrm{c} 1 \mathrm{ccc}(\mathrm{c}(\mathrm{c} 1) \mathrm{OC}) \mathrm{OC}) \mathrm{c} 1 \mathrm{ccccc} 1$ & 699.831 & 9 & 1 & 3.4183 & 10 \\
\hline 11_6w.mol & COc1ccc $(\mathrm{cc} 1) \mathrm{C} 1=\mathrm{CC}(=\mathrm{Nc} 2 \mathrm{cc}(\mathrm{nn} 21) \mathrm{c} 1 \mathrm{ccccc} 1) \mathrm{C}(=\mathrm{O}) \mathrm{N} 1 \mathrm{CCN}(\mathrm{CC} 1) \mathrm{C}(=\mathrm{O}) \mathrm{c} 1 \mathrm{cccnc} 1 \mathrm{Nc} 1 \mathrm{cc}(\mathrm{c}(\mathrm{c}(\mathrm{c} 1) \mathrm{OC}) \mathrm{OC}) \mathrm{OC}$ & 699.831 & 9 & 1 & 3.4183 & 10 \\
\hline 11_6q.mol & COc1ccc $(\operatorname{cc} 1 \mathrm{OC}) \mathrm{Nc} 1 \mathrm{ncccc} 1 \mathrm{C}(=\mathrm{O}) \mathrm{N} 1 \mathrm{CCN}(\mathrm{CC} 1) \mathrm{C}(=\mathrm{O}) \mathrm{C} 1=\mathrm{Nc} 2 \mathrm{cc}(\mathrm{nn} 2 \mathrm{C}(=\mathrm{C} 1) \mathrm{c} 1 \mathrm{ccc}(\mathrm{c}(\mathrm{c} 1) \mathrm{Cl}) \mathrm{Cl}) \mathrm{c} 1 \mathrm{ccccc} 1$ & 708.651 & 7 & 1 & 4.9597 & 8 \\
\hline 11_6t.mol & COc1ccc(cc1OC)Nc1nccc1C(=O)N1CCN(CC1)C(=O)C1=Nc2cc(nn2C(=C1)c1cc(c(c(c1)OC)OC)OC)c1cccc1 & 729.861 & 10 & 1 & 3.1656 & 11 \\
\hline 11_6x.mol & COc1ccc $(\operatorname{cc} 1 \mathrm{OC}) \mathrm{C} 1=\mathrm{CC}(=\mathrm{Nc} 2 \mathrm{cc}(\mathrm{nn} 21) \mathrm{c} 1 \mathrm{ccccc} 1) \mathrm{C}(=\mathrm{O}) \mathrm{N} 1 \mathrm{CCN}(\mathrm{CC} 1) \mathrm{C}(=\mathrm{O}) \mathrm{c} 1 \mathrm{cccnc1N} c 1 \mathrm{cc}(\mathrm{c}(\mathrm{c}(\mathrm{c} 1) \mathrm{OC}) \mathrm{OC}) \mathrm{OC}$ & 729.861 & 10 & 1 & 3.1656 & 11 \\
\hline 11_6v.mol & COc1cc(cc(c1OC)OC)Nc1nccc1C $(=\mathrm{O}) \mathrm{N} 1 \mathrm{CCN}(\mathrm{CC} 1) \mathrm{C}(=\mathrm{O}) \mathrm{C} 1=\mathrm{Nc} 2 \mathrm{cc}(\mathrm{nn} 2 \mathrm{C}(=\mathrm{C} 1) \mathrm{c} 1 \mathrm{ccc}(\mathrm{c}(\mathrm{c} 1) \mathrm{Cl}) \mathrm{Cl}) \mathrm{c} 1 \mathrm{cccc} 1$ & 738.681 & 8 & 1 & 4.707 & 9 \\
\hline
\end{tabular}

Table 2: Compounds which exhibited better binding affinities than bound tamoxifen

\begin{tabular}{|l|l|r|}
\hline S. No & Ligand & MolDock Score \\
\hline 1 & $11 \_6 j . m o l$ & -175.282 \\
\hline 2 & $11 \_6 o . m o l$ & -172.882 \\
\hline 3 & $14 \_16 c . m o l$ & -171.234 \\
\hline 4 & $11 \_6 h . m o l$ & -169.719 \\
\hline 5 & $14 \_15 e . m o l$ & -168.139 \\
\hline 6 & $11 \_6 e . m o l$ & -167.14 \\
\hline
\end{tabular}

\begin{tabular}{|l|l|r|}
\hline 7 & $14 \_16 d . m o l$ & -165.673 \\
\hline 8 & $11 \_6 g . m o l$ & -165.019 \\
\hline 9 & $14 \_15 c . m o l$ & -164.805 \\
\hline 10 & $11 \_6 \mathrm{k} . m o l$ & -164.018 \\
\hline 11 & $14 \_18 \mathrm{e} . \mathrm{mol}$ & -162.91 \\
\hline 12 & $11 \_6 \mathrm{~d} . \mathrm{mol}$ & -162.147 \\
\hline 13 & $11 \_6 \mathrm{t} . \mathrm{mol}$ & -161.625 \\
\hline 14 & $14 \_18 f . m o l$ & -160.374 \\
\hline
\end{tabular}




\begin{tabular}{|c|c|c|}
\hline 15 & 14_18d.mol & -159.463 \\
\hline 16 & 11_6n.mol & -158.725 \\
\hline 17 & 11_6i.mol & -158.478 \\
\hline 18 & 14_18a.mol & -157.811 \\
\hline 19 & 11_6a.mol & -156.748 \\
\hline 20 & 11_6c.mol & -156.4 \\
\hline 21 & 11_6s.mol & -156.129 \\
\hline 22 & 11_6q.mol & -156.068 \\
\hline 23 & 11_6p.mol & -155.936 \\
\hline 24 & 11_6f.mol & -155.614 \\
\hline 25 & 11_6b.mol & -154.125 \\
\hline 26 & 14_15a.mol & -154.078 \\
\hline 27 & 11_6x.mol & -153.768 \\
\hline 28 & 11_61.mol & -153.718 \\
\hline 29 & 14_18b.mol & -153.633 \\
\hline 30 & 11_6m.mol & -153.504 \\
\hline 31 & 14_16a.mol & -152.413 \\
\hline 32 & Tamoxifen & -149.856 \\
\hline
\end{tabular}

\section{Consensus scoring for enrichment of drugs:}

In general, docking routines have the capability to correctly predict protein-ligand complex structures with rational accuracy which is determined based on the RMSD of docked ligand within active site space of the target protein. The ability to forecast the possible geometric binding mode of the docked ligand to distinguish exact poses from incorrect ones is dependent on various scoring functions. Therefore, as is evidenced that both docking analysis and scoring functions play vital importance in drug design procedures, it was reported that the weakness of docking programs is their built-in scoring functions. The main scoring functions include the knowledge-based [36], Physics-based [37], and empirical [38] scoring functions. Therefore, combining various scoring functions would certainly minimize the errors that appear in single scoring programs and thereby enhance the chance of recognizing true hits [39]. Thus, it has been demonstrated that consensus scoring is generally more effective than single scoring for molecular docking [40] and represented an effective way in getting improved hit rates in various virtual database screening studies [41]. In this study, about five scoring functions were employed to evaluate consensus scoring patterns, they are: MolDock score of Molegro, Swiss Dock, mcule docking paradigm, Pose \& Rank scoring, DSX scoring schemes respectively. Classes were generated based on the dock scores followed by ranking the best conformations.

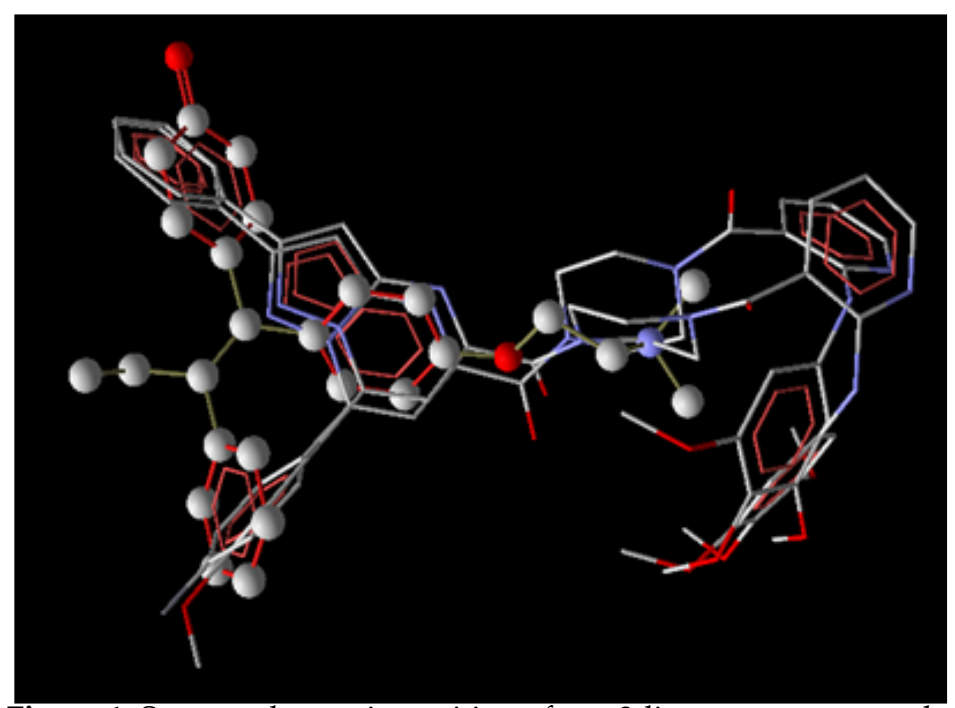

Figure 1: Structural superimposition of top 3 literature compounds Vstamoxifen (ball and stick model).

\section{Results and Discussion:}

The crystal structure of human estrogen receptor alpha ligand binding domain in complex with 4-hydroxytamoxifen (PDB ID: 3ERT) was used for the docking. A thorough analysis of the X-ray crystal structure of estrogen receptor revealed that the active site regions has flexible amino acid side chains and hence could accommodate different chemical scaffolds. The amino acid residues lining active site are: Phe404, Glu419, Leu428, Met343, Gly420, Met421, Leu525, Gly521, Thr347, Leu387, Asp351, Ala350, Glu353, Trp383, Arg394, Leu346, respectively. The protein was prepared using Molegro software. All bond orders and hybridization were assigned, hydrogen and other missing atoms were added to the residues and charges were assigned. The co-crystallized water molecules were excluded from docking. Cavities in the protein were evaluated by Cavity detection algorithm using Expanded Van der Waals molecular surface with default parameters such as minimum and maximum cavity volume set at 10 and $10000 \AA$, with $1.20 \AA$ probe radius and grid resolution being 0.80 resulted in 5 cavities. A docking template was created using bound ligand, with a probe radius of $1.20 \AA$ is used as template for docking external ligands within the active site space of protein. In this case, tamoxifen co-crystallized in ER $\alpha$ was set as ligand template and docking routine was performed using this template complexed in first cavity. 3ERT subjected to docking in triplicate in silico analysis using default parameters of Molegro resulted in RMSD less than $2 \AA$ 
in all cases with average dock score $-149.856 \mathrm{kcal} / \mathrm{mol}$ and RMSD $0.85 \AA$.

\section{Set-1: ERa ligands from literature}

All 105 literature compounds (Table 1) converted into 3D formats are subjected to docking against ER $\alpha$ protein 3ERT using default parameters. Docking analysis resulted in varied dock scores, and compounds that exhibited better binding affinities than tamoxifen are given in Table 2. From Table 2, it is evidenced that nearly 31 compounds displayed better binding affinities than 3ERT bound tamoxifen $(-149.856 \mathrm{kcal} / \mathrm{mol})$. The maximum score obtained was $175.282 \mathrm{kcal} / \mathrm{mol}$ for compound 11_6j. Interestingly, almost all compounds under 11 and 14 series displayed better affinities than tamoxifen. Compounds under 11 series represent pyrazolo[1,5a]pyrimidine analogs whereas 3-aryl-4-anilino-2H-chromen-2-ones were reported under 14 series. The superimposed structures of top 3 compounds with tamoxifen are given in Figure $\mathbf{1}$ and the h-bond interactions are given in Table 3.

An electrostatic interaction was observed when the ligand interacted with oxygen atoms of Asp351. On the other hand, all other interacting amino acids displayed H-bond forces. Further, careful observations on the interacting amino acid residues revealed that pyrazolo[1,5-a]pyrimidine analogs under 11 series displayed major interactions with Thr347 whereas the 3-aryl-4anilino-2H-chromen-2-ones reported under 14 series interacted majorly with His524 amino acid. The ER $\alpha$ bound tamoxifen displayed favourable interactions with Asp351 and Arg394, respectively. Similar interactions are observed with majority of the 14 series chromene derivatives.

\section{Set-2: ERa Non-tested ligands from literature}

A thorough literature search was made on structural features of ligands that would fit into the active site region of $E R \alpha$, which resulted in pyrazole, bipyrazole, thiazole, thiadiazoleetc scaffold analogs. Bipyrazoles are known to possess inhibitory properties against several classes of enzymes. Moreover, preliminary docking analysis revealed better inhibition of ER $\alpha$ with bipyrazoles. Other classes of compounds displayed reduced inhibition. Hence, bipyrazoles are considered for further analysis.

\section{Computational molecular docking and structural specificity of bipyrazoles as inhibitors of ERo}

Docking of all 34 bipyrazoles from literature was carried out to evaluate the best conformer based on the lowest docked energy (kcal/mol) (Table 4), in other words, it should possess highest affinity towards the binding site [42].

From the bipyrazole Vs ER $\alpha$ docking analysis output, it is evidenced that the bipyrazoles are able to bind and fit into the geometrical space provided by the active site region of $\operatorname{ER} \alpha$. The binding orientations of all bipyrazoles were similar to the cocrystallized ligand, tamoxifen (Figure 2). The best compound 2 (ethyl 5-amino-1-(5-amino-3-anilino-4-ethoxycarbonyl-pyrazol-1-yl)-3anilino-pyrazole-4-carboxylate) from Table-7 displayed a score of $175.9 \mathrm{kcal} / \mathrm{mol}$ which is much better than the ER $\alpha$ bound ligand ($149.8 \mathrm{kcal} / \mathrm{mol})$. A favourable H-bond was formed with Thr347 (Figure 3) as observed with chromene derivatives. The next best compound 29 resulted in dock score $(-167.1 \mathrm{kcal} / \mathrm{mol})$, however two favourable $\mathrm{H}$-bonds were found to interact with compound 29, via Thr347 (Figure 4).

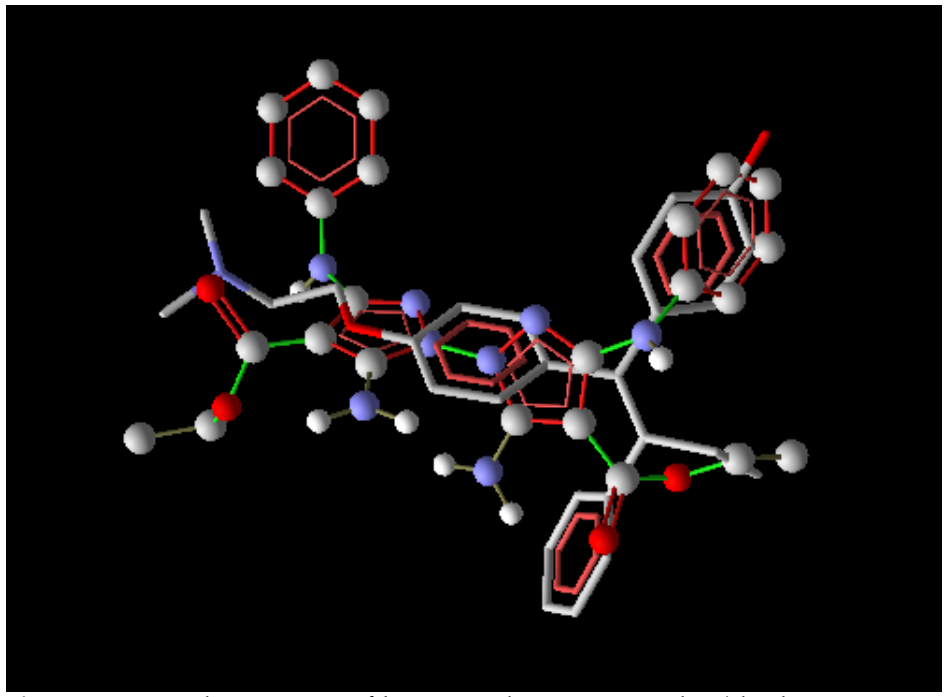

Figure 2: Overlap image of bipyrazole compound 2 (dock score $175.937 \mathrm{kcal} / \mathrm{mol}$ ) with ER $\alpha$ bound tamoxifen. 


\section{BIOINFORMATION \\ Discovery at the interface of physical and biological sciences}

Table 3: H-bond interactions of top 31 compounds

\begin{tabular}{|c|c|c|c|c|}
\hline S. No & Ligand & MolDock Score & H-bond interacting amino acid residues & H-bond energy (kcal/mol) \\
\hline 1 & 11_6j.mol & -175.282 & Cys530, Thr347 & -4.617 \\
\hline 2 & 11_6o.mol & -172.882 & Cys530, Thr347 & -5.0 \\
\hline 3 & 14_16c.mol & -171.234 & Arg394, Glu353, His524 & -5.644 \\
\hline 4 & 11_6h.mol & -169.719 & Thr347 & -0.200 \\
\hline 5 & 14_15e.mol & -168.139 & Arg394, Glu353, His524 & -5.336 \\
\hline 6 & 11_6e.mol & -167.14 & Thr347, Asp351 & -3.020 \\
\hline 7 & 14_16d.mol & -165.673 & Arg394, His524 & -3.141 \\
\hline 8 & 11_6g.mol & -165.019 & Thr347 & -2.455 \\
\hline 9 & 14_15c.mol & -164.805 & His524 & -1.214 \\
\hline 10 & 11_6k.mol & -164.018 & Thr347 & -2.059 \\
\hline 11 & 14_18e.mol & -162.91 & Arg394, Glu353, His524 & -5.729 \\
\hline 12 & 11_6d.mol & -162.147 & Cys530, Thr347 & -2.789 \\
\hline 13 & 11_6t.mol & -161.625 & Leu536, Thr347, His524 & -4.251 \\
\hline 14 & 14_18f.mol & -160.374 & Arg394, Glu353, His524 & -5.331 \\
\hline 15 & 14_18d.mol & -159.463 & Arg394, His524 & -3.600 \\
\hline 16 & 11_6n.mol & -158.725 & Thr347 & -2.333 \\
\hline 17 & 11_6i.mol & -158.478 & Thr347 & -2.388 \\
\hline 18 & 14_18a.mol & -157.811 & Arg394, Glu353, His524, Leu387, Asp351 & -6.595 \\
\hline 19 & 11_6a.mol & -156.748 & Thr347 & -2.500 \\
\hline 20 & 11_6c.mol & -156.4 & Thr347 & -2.369 \\
\hline 21 & 11_6s.mol & -156.129 & Thr347, Cys530 & -3.623 \\
\hline 22 & 11_6q.mol & -156.068 & Thr347 & -2.227 \\
\hline 23 & 11_6p.mol & -155.936 & Thr347 & -1.414 \\
\hline 24 & 11_6f.mol & -155.614 & Thr347 & -2.500 \\
\hline 25 & 11_6b.mol & -154.125 & Thr347 & -1.064 \\
\hline 26 & 14_15a.mol & -154.078 & Asp351, His524 & -1.176 \\
\hline 27 & 11_6x.mol & -153.768 & Arg394 & -1.237 \\
\hline 28 & 11_61.mol & -153.718 & Thr347, Cys530 & -2.682 \\
\hline 29 & 14_18b.mol & -153.633 & Glu353, Arg394, His524 & -5.869 \\
\hline 30 & 11_6m.mol & -153.504 & Thr347 & -2.073 \\
\hline 31 & 14_16a.mol & -152.413 & Asp351, Arg394, His524 & -3.214 \\
\hline 32 & Tamoxifen & -149.856 & Asp351, Arg394 & -2.500 \\
\hline
\end{tabular}

Table 4: IUPAC names, SMILES notation and molecular dock scores in $\mathrm{kcal} / \mathrm{mol}$ of 34 bipyrazole class of compounds.

\begin{tabular}{|c|c|c|c|}
\hline ID & IUPAC Name & SMILES & $\begin{array}{r}\text { Dock Score } \\
(\mathrm{kcal} / \mathrm{mol})\end{array}$ \\
\hline 1 & ethyl 5-amino-3-anilino-1H-pyrazole-4-carboxylate & $\mathrm{CCOC}(=\mathrm{O}) \mathrm{c} 1 \mathrm{c}(\mathrm{N})[\mathrm{nH}] \mathrm{nc} 1 \mathrm{Nc} 2 \mathrm{ccccc} 2$ & -105.689 \\
\hline 2 & $\begin{array}{l}\text { ethyl 5-amino-1-(5-amino-3-anilino-4-ethoxycarbonyl-pyrazol-1-yl)-3-anilino- } \\
\text { pyrazole-4-carboxylate }\end{array}$ & $\mathrm{CCOC}(=\mathrm{O}) \mathrm{c} 1 \mathrm{c}(\mathrm{N}) \mathrm{n}(\mathrm{nc} 1 \mathrm{Nc} 2 \mathrm{ccccc} 2) \mathrm{n} 3 \mathrm{nc}(\mathrm{Nc} 4 \operatorname{ccccc} 4) \mathrm{c}(\mathrm{C}(=\mathrm{O}) \mathrm{OCC}) \mathrm{c} 3 \mathrm{~N}$ & -175.937 \\
\hline 4 & $\begin{array}{l}\text { 5-(4-chlorophenyl)-4-(4-cyanopyrazol-1-yl)-N-(4-phenylphenyl)-3,4- } \\
\text { dihydropyrazole-2-carboxamide }\end{array}$ & $\operatorname{Clc} 1 \mathrm{ccc}(\mathrm{cc} 1) \mathrm{C} 2=\mathrm{NN}(\mathrm{CC} 2 \mathrm{n} 3 \mathrm{cc}(\mathrm{cn} 3) \mathrm{C} \# \mathrm{~N}) \mathrm{C}(=\mathrm{O}) \mathrm{Nc} 4 \mathrm{ccc}(\mathrm{cc} 4) \mathrm{c} 5 \mathrm{ccccc} 5$ & -139.765 \\
\hline 5 & 1-(1,5-diphenylpyrazol-4-yl)-3,5-dimethyl-pyrazole & $\operatorname{Cc} 1 \mathrm{cc}(\mathrm{C}) \mathrm{n}(\mathrm{n} 1) \operatorname{c} 2 \operatorname{cnn}(\operatorname{c} 3 \operatorname{ccc} c \mathrm{c} 3) \operatorname{coc} 4 \operatorname{ccccc} 4$ & -131.507 \\
\hline 7 & 1-tert-butyl-4-(3,5-dimethylpyrazol-1-yl)-5-phenyl-pyrazole & Cc1cc $(\mathrm{C}) \mathrm{n}(\mathrm{n} 1) \mathrm{c} 2 \mathrm{cnn}(\mathrm{c} 2 \mathrm{c} 3 \operatorname{ccccc} 3) \mathrm{C}(\mathrm{C})(\mathrm{C}) \mathrm{C}$ & -117.359 \\
\hline 8 & $\begin{array}{l}\text { bis(2-adamantyl)-[2-[1-(4-methoxyphenyl)-3,5-diphenyl-pyrazol-4-yl]pyrazol-3- } \\
\text { yl]phosphane }\end{array}$ & $\begin{array}{l}\text { COc1ccc(cc1)n2nc(c3cccc3)c(c2c4ccccc4)n5nccc5P(C6C7CC8CC(CC6C8)C7) } \\
\text { C9C\%10CC\%11CC(СС9C\%11) C\%10 }\end{array}$ & -146.054 \\
\hline 9 & $\begin{array}{l}\text { dicyclohexyl-[2-[1-(4-methoxyphenyl)-3,5-diphenyl-pyrazol-4-yl]pyrazol-3- } \\
\text { yl]phosphane }\end{array}$ & COc1ccc(cc1)n2nc(c3ccccs3)c(c2c4ccccc4)n5nccc5P (С6CСССС6)C7CCССC7 & -148.556 \\
\hline 10 & $\begin{array}{l}\text { ditert-butyl-[2-[1-(4-methoxyphenyl)-3,5-diphenyl-pyrazol-4-yl]pyrazol-3- } \\
\text { yl]phosphane }\end{array}$ & $\operatorname{COc} 1 \operatorname{ccc}(\operatorname{cc} 1) n 2 n c(\operatorname{cosccc} 3) \mathrm{c}(\mathrm{c} 2 \operatorname{cocccc} 4) \mathrm{n} 5 \operatorname{nccc} 5 \mathrm{P}(\mathrm{C}(\mathrm{C})(\mathrm{C}) \mathrm{C}) \mathrm{C}(\mathrm{C})(\mathrm{C}) \mathrm{C}$ & -147.159 \\
\hline 11 & 4-chloro-1-(3,5-dinitro-1H-pyrazol-4-yl)-5-nitro-pyrazole & {$[\mathrm{O}-][\mathrm{N}+](=\mathrm{O}) \mathrm{c} 1 \mathrm{n}[\mathrm{nH}] \mathrm{c}(\mathrm{c} 1 \mathrm{n} 2 \mathrm{ncc}(\mathrm{Cl}) \mathrm{c} 2[\mathrm{~N}+](=\mathrm{O})[\mathrm{O}-])[\mathrm{N}+](=\mathrm{O})[\mathrm{O}-]$} & -110.157 \\
\hline 12 & 1-(3,5-dinitro-1H-pyrazol-4-yl)-4,5-dinitro-pyrazole & {$[\mathrm{O}-][\mathrm{N}+](=\mathrm{O}) \mathrm{c} 1 \mathrm{cnn}(\mathrm{c} 1[\mathrm{~N}+](=\mathrm{O})[\mathrm{O}-]) \mathrm{c} 2 \mathrm{c}(\mathrm{n}[\mathrm{nH}] \mathrm{c} 2[\mathrm{~N}+](=\mathrm{O})[\mathrm{O}-])[\mathrm{N}+](=\mathrm{O})[\mathrm{O}-]$} & -117.658 \\
\hline 13 & 1-methyl-3,4-dinitro-5-(3-nitropyrazol-1-yl)pyrazole & $\operatorname{Cn} 1 n c(c(c 1 n 2 \operatorname{ccc}(n 2)[\mathrm{N}+](=\mathrm{O})[\mathrm{O}-])[\mathrm{N}+](=\mathrm{O})[\mathrm{O}-])[\mathrm{N}+](=\mathrm{O})[\mathrm{O}-]$ & -109.876 \\
\hline 15 & $\begin{array}{l}\text { N-[1-(4-methoxyphenyl)-3-methyl-5-pyrazol-1-yl-pyrazol-4- } \\
\text { yl]methanesulfonamide }\end{array}$ & $\operatorname{COc} 1 \mathrm{ccc}(\mathrm{cc} 1) \mathrm{n} 2 \mathrm{nc}(\mathrm{C}) \mathrm{c}(\mathrm{NS}(=\mathrm{O})(=\mathrm{O}) \mathrm{C}) \mathrm{c} 2 \mathrm{n} 3 \mathrm{cccn} 3$ & -125.453 \\
\hline 16 & $\begin{array}{l}\mathrm{N}-[1-(4-\text { bromophenyl)-3-methyl-5-pyrazol-1-yl-pyrazol-4- } \\
\text { yl]methanesulfonamide }\end{array}$ & Cc1nn $(\mathrm{c} 2 \mathrm{ccc}(\mathrm{Br}) \mathrm{cc} 2) \mathrm{c}(\mathrm{c} 1 \mathrm{NS}(=\mathrm{O})(=\mathrm{O}) \mathrm{C}) \mathrm{n} 3 \mathrm{ccc} 3$ & -120.706 \\
\hline
\end{tabular}




\section{BIOINFORMATION \\ Discovery at the interface of physical and biological science.}

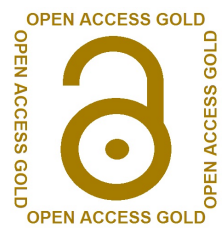

\begin{tabular}{|c|c|}
\hline 17 & $\begin{array}{l}\text { N-[1-(4-chlorophenyl)-3-methyl-5-pyrazol-1-yl-pyrazol-4- } \\
\text { yl]methanesulfonamide }\end{array}$ \\
\hline 18 & $\begin{array}{l}\text { N-[1-(4-fluorophenyl)-3-methyl-5-pyrazol-1-yl-pyrazol-4- } \\
\text { yl]methanesulfonamide }\end{array}$ \\
\hline 19 & $\begin{array}{l}\text { N-[3-methyl-1-(4-nitrophenyl)-5-pyrazol-1-yl-pyrazol-4- } \\
\text { yl]methanesulfonamide }\end{array}$ \\
\hline 20 & $\begin{array}{l}\text { ethyl 5-amino-1-(5-methyl-4-nitro-2-phenyl-pyrazol-3-yl)pyrazole-4- } \\
\text { carboxylate }\end{array}$ \\
\hline 21 & $\begin{array}{l}\text { 3-acetyl-1-(4-bromo-3-phenyl-1H-pyrazol-5-yl)-5-phenyl-pyrazole-4- } \\
\text { carbonitrile }\end{array}$ \\
\hline 22 & $\begin{array}{l}\text { ethyl 3-acetyl-5-amino-1-(4-bromo-3-phenyl-1H-pyrazol-5-yl)pyrazole-4- } \\
\text { carboxylate }\end{array}$ \\
\hline 23 & 1-(4-nitrophenyl)-3-[1-(4-nitrophenyl)-5-propyl-pyrazol-3-yl]-5-propyl-pyrazole \\
\hline 24 & $\begin{array}{l}\text { 5-isopropyl-3-[5-isopropyl-1-(4-nitrophenyl)pyrazol-3-yl]-1-(4- } \\
\text { nitrophenyl)pyrazole }\end{array}$ \\
\hline 25 & $\begin{array}{l}\text { 5-[5-carbamoyl-1-(2,4-dichlorophenyl)-4H-pyrazol-3-yl]-2-(2,4- } \\
\text { dichlorophenyl)pyrazole-3-carboxamide }\end{array}$ \\
\hline 26 & $\begin{array}{l}\text { 2-[5-[5-(1,3-benzothiazol-2-yl)-1,4-bis(4-chlorophenyl)pyrazol-3-yl]-2,4-bis(4- } \\
\text { chlorophenyl)-4H-pyrazol-3-yl]-1,3-benzothiazole }\end{array}$ \\
\hline 27 & $\begin{array}{l}\text { [2-(4-chlorophenyl)-5-[1-(4-chlorophenyl)-5-(2-hydroxybenzoyl)-4-phenyl-4H- } \\
\text { pyrazol-3-yl]-4-phenyl-pyrazol-3-yl]-(2-hydroxyphenyl)methanone }\end{array}$ \\
\hline 28 & 1-(4-chlorophenyl)-5-phenyl-3-(1H-pyrazol-3-yl)pyrazole-4-carbohydrazide \\
\hline 29 & $\begin{array}{l}\text { 4-[(4Z)-5-amino-4-[(4-bromophenyl)methylene]pyrazol-3-yl]-1,5-dimethyl-2- } \\
\text { phenyl-pyrazol-3-one }\end{array}$ \\
\hline 30 & (E)-3-(2-hydroxyphenyl)-1-[1-phenyl-3-(2-thienyl)pyrazol-4-yl]prop-2-en-1-one \\
\hline 31 & $\begin{array}{l}\text { 5-methyl-4-[5-(4-oxochromen-3-yl)-4,5-dihydro-1H-pyrazol-3-yl]-1,2- } \\
\text { dihydropyrazol-3-one }\end{array}$ \\
\hline 32 & $\begin{array}{l}\text { 5-amino-N-(1,3-benzothiazol-2-yl)-3-(1,3-diphenylpyrazol-4-yl)-1H-pyrazole-4- } \\
\text { carboxamide }\end{array}$ \\
\hline 33 & 3-(5-hydroxy-3-methyl-1-phenyl-pyrazol-4-yl)-1H-pyrazole-5-carbohydrazide \\
\hline 34 & $\begin{array}{l}\text { diethyl 2-(4-bromophenyl)-5-(4-cyano-5-methyl-2-phenyl-pyrazol-3- } \\
\text { yl)pyrazole-3,4-dicarboxylate }\end{array}$ \\
\hline
\end{tabular}

\begin{tabular}{|c|c|}
\hline Cc1nn $(\mathrm{c} 2 \operatorname{ccc}(\mathrm{Cl}) \operatorname{cc} 2) \mathrm{c}(\mathrm{c} 1 \mathrm{NS}(=\mathrm{O})(=\mathrm{O}) \mathrm{C}) \mathrm{n} 3 \mathrm{ccc} 3$ & -118.696 \\
\hline Cc1nn $(\mathrm{c} 2 \operatorname{ccc}(\mathrm{F}) \mathrm{cc} 2) \mathrm{c}(\mathrm{c} 1 \mathrm{NS}(=\mathrm{O})(=\mathrm{O}) \mathrm{C}) \mathrm{n} 3 \operatorname{ccc} 3$ & -123.955 \\
\hline Cc1nn $(\mathrm{c} 2 \mathrm{ccc}(\mathrm{cc} 2)[\mathrm{N}+](=\mathrm{O})[\mathrm{O}-]) \mathrm{c}(\mathrm{c} 1 \mathrm{NS}(=\mathrm{O})(=\mathrm{O}) \mathrm{C}) \mathrm{n} 3 \mathrm{cccn} 3$ & -121.433 \\
\hline $\operatorname{CCOC}(=\mathrm{O}) \mathrm{c} 1 \mathrm{cnn}(\mathrm{c} 1 \mathrm{~N}) \mathrm{c} 2 \mathrm{c}(\mathrm{c}(\mathrm{C}) \mathrm{nn} 2 \mathrm{c} 3 \operatorname{ccccc} 3)[\mathrm{N}+](=\mathrm{O})[\mathrm{O}-]$ & -133.582 \\
\hline $\mathrm{CC}(=\mathrm{O}) \mathrm{c} 1 \mathrm{nn}(\mathrm{c}(\mathrm{c} 2 \mathrm{ccccc} 2) \mathrm{c} 1 \mathrm{C} \# \mathrm{~N}) \mathrm{c} 3[\mathrm{nH}] \mathrm{nc}(\mathrm{c} 3 \mathrm{Br}) \mathrm{c} 4 \mathrm{ccccc} 4$ & -148.595 \\
\hline $\operatorname{CCOC}(=\mathrm{O}) \mathrm{c} 1 \mathrm{c}(\mathrm{N}) \mathrm{n}(\mathrm{nc} 1 \mathrm{C}(=\mathrm{O}) \mathrm{C}) \mathrm{c} 2[\mathrm{nH}] \mathrm{nc}(\mathrm{c} 2 \mathrm{Br}) \mathrm{c} 3 \mathrm{ccccc} 3$ & -113.874 \\
\hline CCCc1cc(nn1c2ccc(cc2)[N+](=O)[O-])c3cc(CCC)n (n3)c4ccc(cc4)[N+](=O)[O-] & -154.386 \\
\hline $\mathrm{CC}(\mathrm{C}) \mathrm{c} 1 \mathrm{cc}(\mathrm{nn} 1 \mathrm{c} 2 \mathrm{ccc}(\mathrm{cc} 2)[\mathrm{N}+](=\mathrm{O})[\mathrm{O}-]) \mathrm{c} 3 \mathrm{cc}(\mathrm{C}(\mathrm{C}) \mathrm{C}) \mathrm{n}(\mathrm{n} 3) \mathrm{c} 4 \mathrm{ccc}(\mathrm{cc} 4)[\mathrm{N}+](=\mathrm{O})[\mathrm{O}-]$ & -154.361 \\
\hline $\mathrm{NC}(=\mathrm{O}) \mathrm{C} 1=[\mathrm{N}](\mathrm{N}=\mathrm{C}(\mathrm{C} 1) \mathrm{c} 2 \mathrm{cc}(\mathrm{C}(=\mathrm{O}) \mathrm{N}) \mathrm{n}(\mathrm{n} 2) \mathrm{c} 3 \mathrm{ccc}(\mathrm{Cl}) \mathrm{cc} 3 \mathrm{Cl}) \mathrm{c} 4 \mathrm{ccc}(\mathrm{Cl}) \mathrm{cc} 4 \mathrm{Cl}$ & -130.783 \\
\hline $\begin{array}{l}\text { Clc1ccc(cc1)C2C }(=\mathrm{N}[\mathrm{N}](=\mathrm{C} 2 \mathrm{c} 3 \mathrm{nc} 4 \mathrm{ccccc} 4 \mathrm{~s} 3) \mathrm{c} 5 \mathrm{ccc}(\mathrm{Cl}) \mathrm{cc} 5) \mathrm{c} 6 \mathrm{nn}(\mathrm{c} 7 \mathrm{ccc}(\mathrm{Cl}) \mathrm{cc} 7) \mathrm{c}(\mathrm{c} 8 \mathrm{nc} 9 \mathrm{cccc} \mathrm{c} 9 \mathrm{~s} 8) \\
\mathrm{c} 6 \mathrm{c} \% 10 \mathrm{ccc}(\mathrm{Cl}) \mathrm{cc} \% 10\end{array}$ & -138.603 \\
\hline Oc1cccc1C $(=\mathrm{O}) \mathrm{C} 2=[\mathrm{N}](\mathrm{N}=\mathrm{C}(\mathrm{C} 2 \mathrm{c} 3 \operatorname{ccccc} 3) \mathrm{c} 4 \mathrm{nn}(\mathrm{c} 5 \mathrm{ccc}(\mathrm{Cl}) \mathrm{cc} 5) \mathrm{c}(\mathrm{C}(=\mathrm{O}) \operatorname{c6ccccc} 6 \mathrm{O}) \mathrm{c} 4 \mathrm{c} 7 \mathrm{ccccc} 7) \mathrm{c} 8 \mathrm{ccc}(\mathrm{Cl}) \mathrm{cc} 8$ & -140.477 \\
\hline $\mathrm{NNC}(=\mathrm{O}) \mathrm{c} 1 \mathrm{c}(\mathrm{nn}(\mathrm{c} 2 \mathrm{ccc}(\mathrm{Cl}) \mathrm{cc} 2) \mathrm{c} 1 \mathrm{c} 3 \mathrm{ccccc} 3) \mathrm{c} 4 \mathrm{cc}[\mathrm{nH}] \mathrm{n} 4$ & -137.395 \\
\hline $\mathrm{CN} 1 \mathrm{~N}(\mathrm{C}(=\mathrm{O}) \mathrm{C}(=\mathrm{C} 1 \mathrm{C}) \mathrm{C} 2=\mathrm{NN}=\mathrm{C}(\mathrm{N}) / \mathrm{C} / 2=\mathrm{C} \backslash \mathrm{c} 3 \mathrm{ccc}(\mathrm{Br}) \operatorname{cc} 3) \operatorname{c} 4 \mathrm{ccccc} 4$ & -167.179 \\
\hline Oc1ccccc1 $\backslash \mathrm{C}=\mathrm{C} \backslash \mathrm{C}(=\mathrm{O}) \operatorname{c2} \operatorname{cn}(\mathrm{nc} 2 \mathrm{c} 3 \operatorname{cccs} 3) \mathrm{c} 4 \mathrm{ccccc} 4$ & -98.6882 \\
\hline $\mathrm{CC} 1=\mathrm{C}(\mathrm{C}(=\mathrm{O}) \mathrm{NN} 1) \mathrm{C} 2=\mathrm{NNC}(\mathrm{C} 2) \mathrm{C} 3=\mathrm{CO} 4 \operatorname{ccccc} 4 \mathrm{C} 3=\mathrm{O}$ & -138.46 \\
\hline $\mathrm{Nc} 1[\mathrm{nH}] \mathrm{nc}(\mathrm{c} 2 \mathrm{cn}(\mathrm{nc} 2 \mathrm{c} 3 \mathrm{ccccc} 3) \mathrm{c} 4 \mathrm{cccc} 4) \mathrm{c} 1 \mathrm{C}(=\mathrm{O}) \mathrm{Nc} 5 \mathrm{nc} 6 \mathrm{cccc} 6 \mathrm{~s} 5$ & -145.914 \\
\hline $\mathrm{Cc} 1 \mathrm{nn}(\mathrm{c}(\mathrm{O}) \mathrm{c} 1 \mathrm{c} 2 \mathrm{cc}([\mathrm{nH}] \mathrm{n} 2) \mathrm{C}(=\mathrm{O}) \mathrm{NN}) \mathrm{c} 3 \mathrm{ccccc} 3$ & -117.006 \\
\hline $\operatorname{CCOC}(=\mathrm{O}) \mathrm{c} 1 \mathrm{c}(\mathrm{nn}(\mathrm{c} 2 \mathrm{ccc}(\mathrm{Br}) \mathrm{cc} 2) \mathrm{c} 1 \mathrm{C}(=\mathrm{O}) \mathrm{OCC}) \mathrm{c} 3 \mathrm{c}(\mathrm{C \# N}) \mathrm{c}(\mathrm{C}) \mathrm{nn} 3 \mathrm{c} 4 \mathrm{ccccc} 4$ & -121.309 \\
\hline
\end{tabular}

Table 5: Screening result of DrugBank database against ER $\alpha$ showing binding affinities (kcal/mol).

\begin{tabular}{|c|c|c|c|c|c|}
\hline $\begin{array}{l}\text { DrugBa } \\
\text { nk ID }\end{array}$ & $\begin{array}{l}\text { Binding affinity } \\
\text { (kcal/mol) }\end{array}$ & Drug Name & $\begin{array}{l}\text { Interaction } \\
\text { Type }\end{array}$ & $\begin{array}{l}\text { Interacting } \\
\text { Residues }\end{array}$ & Drug Indication, disease and related information \\
\hline DB09065 & -187.123 & Cobicistat & H-bonding & Arg394, Cys530 & Cobicistat is a CYP3A inhibitor \\
\hline DB08827 & -185.233 & Lomitapide & Van der Waals & No interactions & Used in homozygous familial hypercholesterolemia (HoFH) patients \\
\hline DB01167 & -180.646 & Itraconazole & H-bonding & Thr347, Cys530 & For the treatment of the fungal infections \\
\hline DB06809 & -178.689 & Plerixafor & H-bonding & Glu353 & $\begin{array}{l}\text { Used in combination with granulocyte-colony stimulating factor (G-CSF, } \\
\text { filgrastim) in patients with non-Hodgkin's lymphoma (NHL) and multiple } \\
\text { myeloma (MM). }\end{array}$ \\
\hline DB08822 & -173.473 & $\begin{array}{l}\text { Azilsartanmed } \\
\text { oxomil }\end{array}$ & H-bonding & Thr347, His524 & Treatment of hypertension (alone or as an adjunct). \\
\hline DB00549 & -172.426 & Zafirlukast & H-bonding & Thr347 & For the prophylaxis and chronic treatment of asthma. \\
\hline DB06401 & -170.261 & Bazedoxifene & H-bonding & $\begin{array}{l}\text { Gly420, His524, } \\
\text { Leu387, Arg394 }\end{array}$ & Bazedoxifene is a third generation selectiveestrogen receptor modulator (SERM). \\
\hline DB01259 & -169.171 & Lapatinib & H-bonding & Thr347, Asp351 & $\begin{array}{l}\text { Indicated in combination with capecitabine for the treatment of patients with } \\
\text { advanced or metastatic breast cancer }\end{array}$ \\
\hline DB00430 & -166.876 & Cefpiramide & H-bonding & $\begin{array}{l}\text { Thr347, Asp351, } \\
\text { Leu525 }\end{array}$ & $\begin{array}{l}\text { For treatment of severe infections caused by susceptible bacteria such as P. } \\
\text { aeruginosa. }\end{array}$ \\
\hline DB01264 & -165.672 & Darunavir & H-bonding & Leu346, Thr347 & $\begin{array}{l}\text { Darunavir, co-administered with ritonavir is indicated for the treatment of HIV } \\
\text { infection }\end{array}$ \\
\hline DB00503 & -165.18 & Ritonavir & Van der Waals & No interactions & $\begin{array}{l}\text { Indicated in combination with other antiretroviral agents for the treatment of HIV- } \\
1 \text { infection. }\end{array}$ \\
\hline DB01263 & -164.662 & Posaconazole & H-bonding & $\begin{array}{l}\text { Glu353, Leu387, } \\
\text { Cys530 }\end{array}$ & For prophylaxis of invasive Aspergillus and Candida infections \\
\hline DB00481 & -163.664 & Raloxifene & H-bonding & $\begin{array}{l}\text { Arg394, Glu353, } \\
\text { Gly521, Gly420, } \\
\text { His524 }\end{array}$ & $\begin{array}{l}\text { A second generation selective estrogen receptor modulator (SERM), for the } \\
\text { prevention and treatment of osteoporosis in post-menopausal women }\end{array}$ \\
\hline DB08912 & -163.634 & Dabrafenib & H-bonding & Gly521 & Indicated for the treatment of patients with unresectable or metastatic melanoma. \\
\hline DB06590 & -163.214 & $\begin{array}{l}\text { Ceftarolinefosa } \\
\text { mil }\end{array}$ & H-bonding & $\begin{array}{l}\text { Met343, Thr347, } \\
\text { Cys530 }\end{array}$ & Ceftarolinefosamil is a cephalosporin antibacterial. \\
\hline
\end{tabular}


Table 6: DrugBank drugs and corresponding scores of five scoring functions with rank-sum technique

\begin{tabular}{|c|c|c|c|c|c|c|c|c|c|c|c|c|}
\hline Drug Name & DrugBank ID & DSX online & Rank & $\begin{array}{l}\text { Pose\& } \\
\text { Rank }\end{array}$ & Rank & MolDock & Rank & mcule & Rank & $\begin{array}{l}\text { Swiss } \\
\text { Dock }\end{array}$ & Rank & Rank-Sum \\
\hline Cobicistat & DB09065 & -124 & 2 & -52.01 & 3 & -187.123 & 3 & -8 & 1 & -10.08 & 3 & 12 \\
\hline Lomitapide & DB08827 & -166 & 3 & -49.06 & 3 & -185.233 & 3 & -10.3 & 3 & -9.77 & 3 & 15 \\
\hline Itraconazole & DB01167 & -125 & 2 & -44.55 & 3 & -180.646 & 3 & -10.4 & 3 & -9.52 & 3 & 14 \\
\hline Plerixafor & DB06809 & -105 & 1 & -25.77 & 1 & -178.689 & 2 & -9.6 & 3 & -9.87 & 3 & 10 \\
\hline Azilsartanmedoxomil & DB08822 & -107 & 2 & -42.56 & 2 & -173.473 & 2 & -9.7 & 3 & -9.06 & 3 & 12 \\
\hline Zafirlukast & DB00549 & -137 & 3 & -46.05 & 3 & -172.426 & 2 & -9.3 & 2 & -8.36 & 2 & 12 \\
\hline Cefpiramide & DB00430 & -96 & 1 & -31.8 & 1 & -166.876 & 1 & -8.2 & 1 & -6.83 & 1 & 5 \\
\hline Darunavir & DB01264 & -119 & 2 & -42.25 & 2 & -165.672 & 1 & -8.1 & 1 & -7.82 & 1 & 7 \\
\hline Ritonavir & DB00503 & -74 & 1 & -26.46 & 1 & -165.18 & 1 & -7.6 & 1 & -7.17 & 1 & 5 \\
\hline Posaconazole & DB01263 & -116 & 2 & -26.43 & 1 & -164.662 & 1 & -7.9 & 1 & -7.4 & 1 & 6 \\
\hline Ceftarolinefosamil & DB06590 & -102 & 1 & -25.22 & 1 & -163.214 & 1 & -7.2 & 1 & -7.72 & 1 & 5 \\
\hline
\end{tabular}

DSX, Pose \& Rank, MolDock, mcule, SwissDock values are in kcal/mol

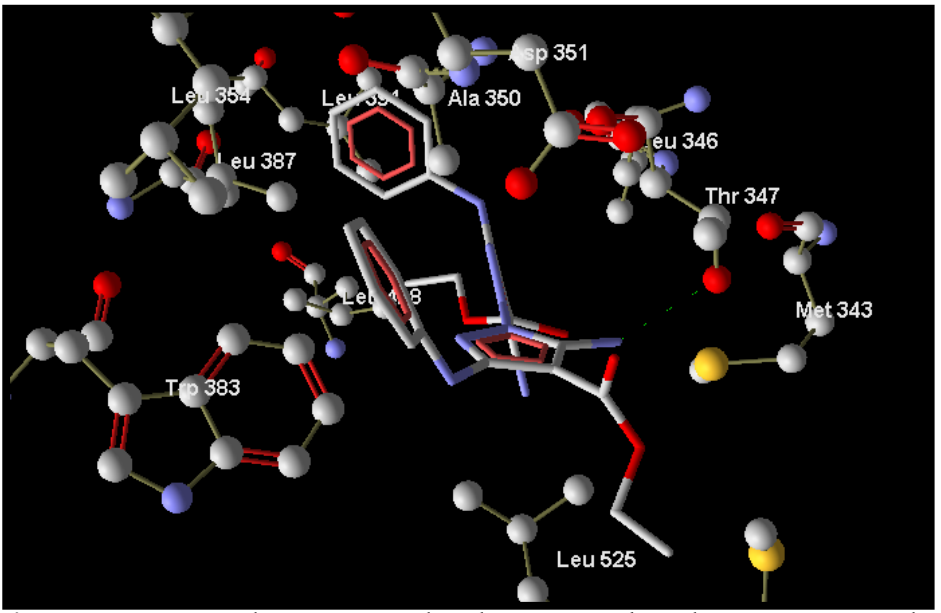

Figure 3: Bipyrazole compound 2 showing H-bond interaction with Thr347.

\section{Set-3: Drugs from DrugBank Database}

Owing to the output from bipyrazole dataset, which showed better inhibitory than tamoxifen, the next step utilized was to search DrugBank database because it was observed that certain drugs which are specific against a particular disease were found to be effective against other disease conditions as well, for example, Pioglitazone, a drug used for type 2 diabetes, may prevent recurrent stroke and heart attacks in people with insulin resistance but without diabetes [43-44]. Several studies indicate that persons with type-2 diabetes are at higher risk of cancer of the pancreas, liver, endometrium, breast, colon, rectum and urinary bladder [45]. however, the use of metformin was associated with decreased risk of the occurrence of various types of cancers, especially of pancreas and colon and hepatocellular carcinoma [46] evidence suggested that metformin might reduce breast cancer incidence in postmenopausal women [47] In another study, by screening already approved drugs, researchers identified calcium channel blockers, which are used to treat hypertension, can efficiently stop cancer cell invasion in vitro [48]. Preliminary investigations revealed that Gleevec blocked the progression and development of rheumatoid arthritis in laboratory mice [49]. Therefore, in this context DrugBank database was accessed to select 2035 FDA approved drugs and subjected to molecular docking. Analysis resulted in 15 drugs, which showed better binding affinities than ER $\alpha$ bound tamoxifen, tabulated in Table 5.

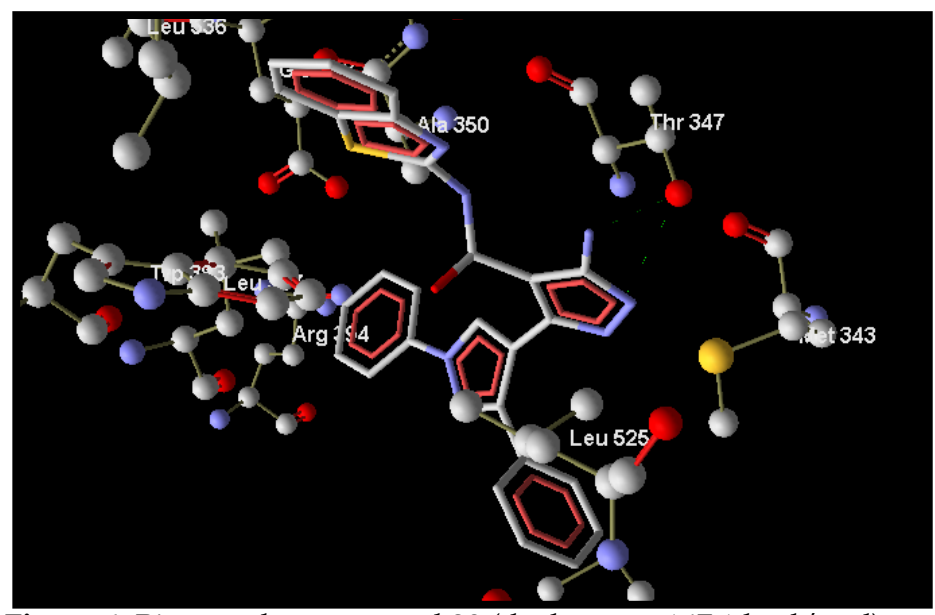

Figure 4: Bipyrazole compound 29 (dock score $-167.1 \mathrm{kcal} / \mathrm{mol}$ ) showing two H-bond interactions with Thr347.

Table 5 represented better inhibitory values of various drugs intended for specific disease conditions when compared to ER $\alpha$ 
bound tamoxifen. The top best compound obtained from analysis was Cobicistat with binding energy, $-187.123 \mathrm{kcal} / \mathrm{mol}$. All drugs displayed H-bond interactions except Lomitapide and Ritonavir, which displayed Van der Waals interactions with $E R \alpha$.Superimposition of all drugs within the active space of ER $\alpha$ is given in Figure 5 where it is evidenced that all drugs occupied clearly within the geometric space of the protein. From the table, out of 15 drugs, only 11 are finalized to consider for further analysis. This is because the four drugs viz., Bazedoxifene, Lapatinib, Raloxifene and Dabrafenib found to be anti-cancer drugs and hence omitted from the list.

\section{Consensus Scoring to enrich drugs active against ERa:}

It has been reported recently that consensus scoring, which combines multiple scoring functions, leads to higher hit-rates in virtual library screening studies [50] and presented an idealized computer experiment to explore how consensus scoring works based on the assumption that the error of a scoring function is a random number in a normal distribution. Many studies suggested that implementing consensus-scoring approaches enhances the performance by compensating for the deficiencies of the scoring functions with each other [51] [52] [53] The possibility that several scoring methods might have their own strengths and weaknesses and combined use of more than one method might increase the overall signal-noise ratio and might perform better than the average of the individual scoring functions [54] presented computer-aided analysis where they implemented an intersection-based consensus approach to group few scoring functions. Stahl and Rarey [55] reported the performance of four scoring functions on seven target proteins.

Screening analysis of DrugBank database drugs against ER $\alpha$ resulted in 11 drugs and all these drugs are subjected to consensus scoring using 5 scoring schemes such as MolDock score of Molegro, mcule, SwissDock, Pose \& Rank and DSX respectively. Here, we chose the "rank-by-number" strategy to pool the output of multiple scoring functions. This is because, this strategy was reported to outperform the other techniques such as "rank-by-rank" and "rankby-vote" as the rank-by-number strategy summarized most of the information [56] Each scoring function was applied to generate three classes based on the obtained dock scores followed by ranking the best conformations. Classes were generated for all scoring functions and instead of taking an average, rank-bynumber technique [57] was employed to finalize best compounds. The ranks obtained from each of the scoring functions were added to give the rank-sum. The benefit of rank-by-number technique is that the each individual score involvement for a rank can certainly be split out for illustrative purposes [58]. The rank sums obtained for 11 drugs against five scoring functions were in the range 5 to 15 , with 5 being low rank and 15 being first and best rank, respectively (Table 6). Therefore, finally from 11 drugs, the top five compounds with rank-sums 15 - 12 (Lomitapide, Itraconazole, Cobicistat, Azilsartanmedoxomil, and Zafirlukast) are finalized. Further work shall be carried out to study their affinity of binding and inhibitory characteristics against ER $\alpha$ in a breast cancer cell line MCF-7.

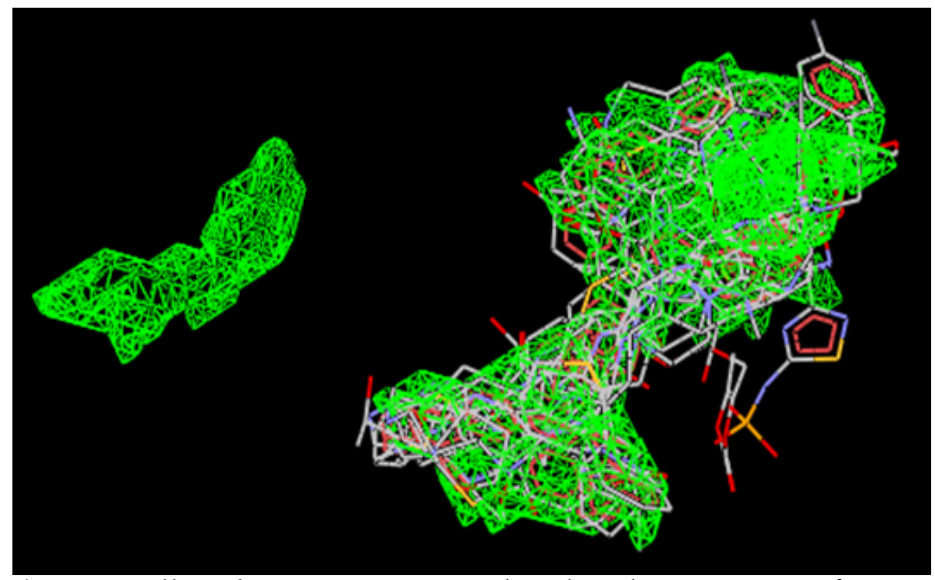

Figure 5: All 15 drugs superimposed within the active site of ER $\alpha$.

\section{Conclusion:}

Molecular docking analysis carried out on a set of ER $\alpha$ inhibitors against 3ERT, complexed with 4-hydroxytamoxifen (-149.856 $\mathrm{kcal} / \mathrm{mol}$ with RMSD $0.85 \AA$ ) resulted in better binding affinities than 3ERT bound tamoxifen for nearly 31 compounds with pyrazolo[1,5-a]pyrimidine and chromen-2-one derivatives. The best compound (-175.282 $\mathrm{kcal} / \mathrm{mol})$ was [2-(4-Fluoro-phenylamino)pyridin-3-yl]-\{4-[2-phenyl-7-(3,4,5-trimethoxy-phenyl)-pyrazolo[1,5-

a]pyrimidine-5-carbonyl]-piperazin-1-yl\}-methanone and favourable interactions were observed with Thr347. In our search to unearth entirely novel compounds, bipyrazole nucleus compounds were analyzed which resulted in with $-175.9 \mathrm{kcal} / \mathrm{mol}$ binding affinity with the receptor and favourable H-bond interaction with Thr347. After realizing this novel inhibitor, 2035 FDA approved drugs from DrugBank database were screened to study their efficacy against $\mathrm{ER} \alpha$, resulted in 15 such drugs with binding affinities greater than tamoxifen ranging from -164.66 to $-187.12 \mathrm{kcal}$ per mol. After eliminating 4 anti-cancer drugs, the remaining 11 drugs are subjected to consensus scoring using MolDock score of Molegro, mcule, SwissDock, Pose\&Rank and DSX. Consensus analysis resulted in top ranks for 5 drugs viz., Lomitapide, Itraconazole, Cobicistat, Azilsartanmedoxomil, and Zafirlukast, which were 


\section{BIOINFORMATION}

selected further to assess their experimental activity in an MCF-7 cell line.

\section{References:}

[1] Clarke R et al. Mol Cell Endocrinol. 2015 418: 220. [PMID: 26455641]

[2] Hefti MM et al. Breast Cancer Research 2013 15:R68 [PMID: 23971947]

[3] Berry DA et al. JAMA. 2006 295:1658 [PMID: 16609087]

[4] Lee HH et al. Endocr Relat Cancer.2008, 15: 765 [PMID: 18469155]

[5] Gross JM \& Yee D. Breast Cancer Research : 2002 4:62[PMID: 11879565]

[6] Fisher B et al. J Natl Cancer Inst 1998 90:1371 [PMID: 9747868]

[7] Kahlert S et al. J Biol Chem. 2000 275:18447 [PMID: 10749889]

[8] Pietras RJ \& Szego CM. Nature 1977 265:69 [PMID: 834244]

[9] Hayes DF et al. Seminars in Oncology 1995 22:5 [PMID: 7537913]

[10] Buzdar AU, Semin Oncol. 2001 28:3 [PMID: 11402439]

[11] Buzdar AU, Seminars in Oncology. 2001 28: 291[PMID: 11402439]

[12] Jaiyesimi IA et al. J ClinOncol. 1995 13:513 [PMID: 7844613]

[13] Ng HWet al. BMC Bioinformatics. 2014 15:S4 [PMID: 25349983]

[14] Nettles KW et al. EMBO Rep. 2007 8:563 [PMID: 17468738]

[15] Bruning JB et al. Nat Chem Biol. 2010 6:837 [PMID: 20924370]

[16] Brooijmans N\& Kuntz ID. Annu Rev Biophys Biomol Struct. 2003 32:335 [PMID: 12574069]

[17] Kroemer RT. Biochemical Society Transactions. 2003 31:980 [PMID: 14505464]

[18] Drews J. Science. 2000 17:287 [PMID: 10720314]

[19] Thomsen R \& Christensen MH. J. Med. Chem. 2006 49:11[PMID: 16722650]

[20] Gasteiger J \& Engel T (2003). Chemo informatics: A Textbook. Wiley-VCH. p. 143 https:// doi.org/10.1002/ cem.871

[21] Schüttelkopf AW \& van Aalten DMF. ActaCrystallogr. 2004 D60 [PMID: 15272157]

[22] Li XY et al. Bioorganic Medicinal Chemistry Letters. 2013 23:16 [PMID: 23830503]

[23] Ohta K et al. Bioorganic Medicinal Chemistry Letters. 2008 18:18 [PMID: 18760603]

[24] Chen XY et al. Bioorganic Medicinal Chemistry Letters. 2012 22:13 [PMID: 22652053]

[25] Haddad T et al. Bioorganic Medicinal Chemistry Letters. 2012 22:18 [PMID: 22906893]

[26] Sala M et al. Bioorganic Medicinal Chemistry Letters. 2013 23:7 [PMID: 23860590]
[27] Archana S et al. Bioorg Med Chem Lett. 2015 25: 680[PMID: 25529734]

[28] Kamal A et al. Bioorganic Medicinal Chemistry Letters. 2016 26:8[PMID: 26948540]

[29] Luo G, Bioorganic Medicinal Chemistry Letters. 2017 27:12[PMID: 28460819]

[30] Rohaiza S et al. J. Pharm. Pharmacol. 2011 5(9), 1272 https://doi.org/10.5897/AJPP10.386

[31] Bioorg RA, Med. Chem. Lett. 2010 20: 22 [PMID: 20851600]

[32] Hu ZF et al. Fitoterapia. 201182:190 [PMID: 20837110]

[33] Abdel-Wahab BF \& Dawood KM ARKIVOC 2012 (i) 491 http:/ / dx.doi.org/10.3998/ark.5550190.0013.112

[34] Bondock S, ARKIVOC 2006 (ix) 113 https:/ / www.arkatusa.org/get-file/ 22751

[35] Wishart DS et al. Nucleic Acids Res. 2017 Nov 8. [PMID: 29126136]

[36] Gohlke H et al. J. Mol. Biol. 2000 295:337 [PMID: 10623530]

[37] Weiner SJ, J. Am. Chem. Soc. 1984 106: 3 https://pubs.acs.org/doi/pdfplus/10.1021/ja00315a051

[38] Gehlhaar DK et al. Chem. Biol. 1995 2: 317 [PMID: 9383433]

[39] Kitchen DB, Nat Rev Drug Discov. 2004 3:935 [PMID: 15520816]

[40] Clark RD, J Mol Graph Modeling 2002 20:281 [PMID: 11858637]

[41] Charifson PS, J Med Chem. 1999 42:5100 [PMID: 10602695]

[42] Azam SS \& Abbasi SW, Theoretical Biology \& Medical Modelling.2013 10:63 [PMID: 24156411]

[43] Kernan WN et al. New England Journal of Medicine. 2016 317:1321 [PMID: 26886418]

[44] Lee M et al. Stroke. 2017 48:388 [PMID: 27999139]

[45] Smith U \& Gale EA. Diabetologia. 2010 53:1541 [PMID: 20549181]

[46] JKasznicki J et al. Ann Transl Med. 2014 2: 57 [PMID: 25333032]

[47] Chlebowski RT et al. J Clin Oncol. 2012 30:2844 [PMID: 22689798]

[48] Jacquemet G et al. Nature Communications 2016 7:13297 [PMCID: PMC5146291]

[49] Paniagua RT et al. J Clin Invest. 2006116:2633 [PMID:16981009]

[50] Wang R \& Wang S. J. Chem. Inf. Comput. Sci. 200141:1422 [PMID: 11604043]

[51] Wang R et al. J Med Chem. 2003 46: 2287[PMID: 12773034]

[52] Bissantz C et al. J Med Chem.2000 43: 4759[PMID: 11123984]

[53] Bissantz C et al. Proteins 2003 50: 5 [PMID: 12471595]

[54] Charifson PS et al. J Med Chem. 1999 42: 5100 [PMID: 10602695] 


\section{BIOINFORMATION \\ Discovery at the interface of physical and biological sciences}

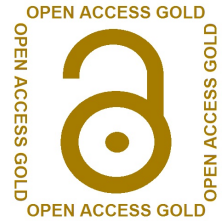

[55] Stahl M \& Rarey M. J. Med. Chem. 2001 44: 1035 [PMID: 11297450]

[57] Ajay MA \& Murcko J. J Med Chem.1995 38:4953 [PMID: 8544170]

[56] Wang R \& Wang S. J. Chem. Inf. Comput. Sci. 2001 41:1422 [PMID: 11604043]

[58] Clark RD, J Mol Graph Modeling2002 20:281 [PMID: 11858637]

Edited by P Kangueane

Citation: Tilak Vijay et al. Bioinformation 15(5): 321-332 (2019)

License statement: This is an Open Access article which permits unrestricted use, distribution, and reproduction in any medium, provided the original work is properly credited. This is distributed under the terms of the Creative Commons Attribution License

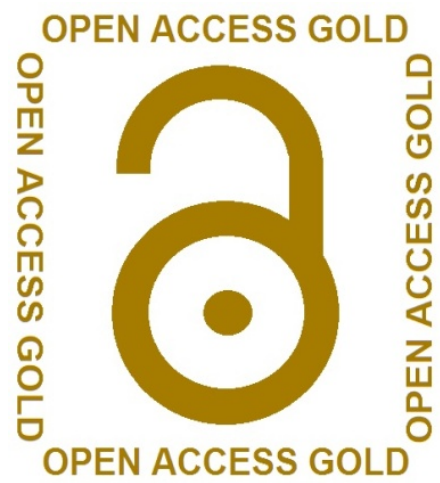

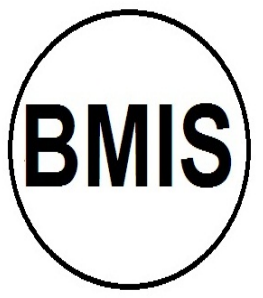

Biomedical Informatics Society

\section{Journal}

